\title{
Orbital dynamics of 'smart dust' devices with solar radiation pressure and drag
}

\author{
Camilla Colombo* and Colin McInnes ${ }^{\dagger}$ \\ University of Strathclyde, Glasgow, UK, G1 1XJ
}

\begin{abstract}
This paper investigates how perturbations due to asymmetric solar radiation pressure, in the presence of Earth shadow, and atmospheric drag can be balanced to obtain long-lived Earth centered orbits for swarms of micro-scale 'smart dust' devices, without the use of active control. The secular variation of Keplerian elements is expressed analytically through an averaging technique. Families of solutions are then identified where Sun-synchronous apse-line precession is achieved passively to maintain asymmetric solar radiation pressure. The long-term orbit evolution is characterized by librational motion, progressively decaying due to the non-conservative effect of atmospheric drag. Long-lived orbits can then be designed through the interaction of energy gain from asymmetric solar radiation pressure and energy dissipation due to drag. In this way, the usual short drag lifetime of such high area-to-mass spacecraft can be greatly extended (and indeed selected). In addition, the effect of atmospheric drag can be exploited to ensure the rapid end-of-life decay of such devices, thus preventing long-lived orbit debris.
\end{abstract}

\section{Nomenclature}

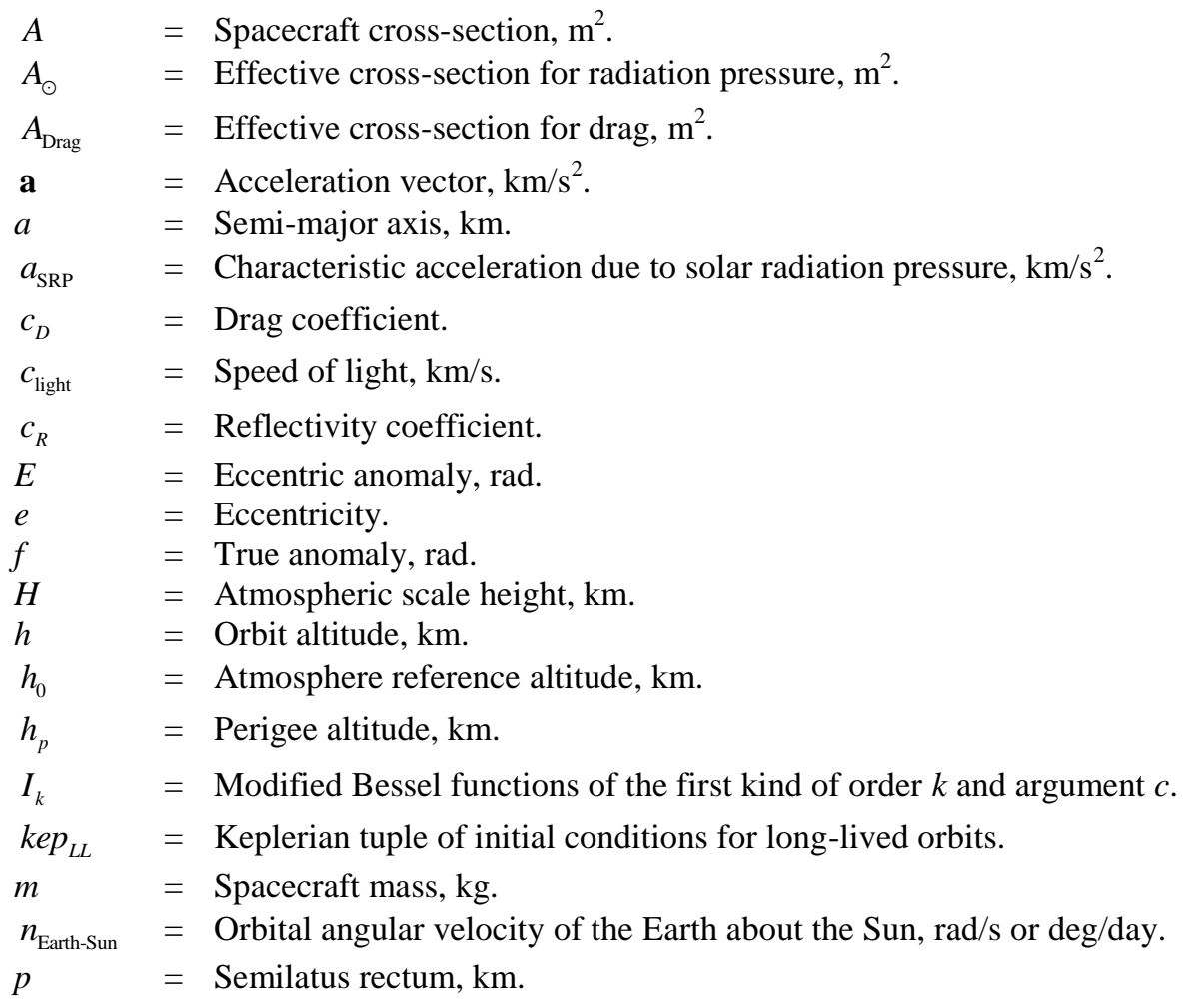

\footnotetext{
"Research Fellow, Advanced Space Concepts Laboratory, Department of Mechanical Engineering, James Weir Building, camilla.colombo@strath.ac.uk; AIAA Member.

${ }^{\dagger}$ Professor, Advanced Space Concepts Laboratory, Department of Mechanical Engineering, James Weir Building, colin.mcinnes@strath.ac.uk; AIAA Member.
} 


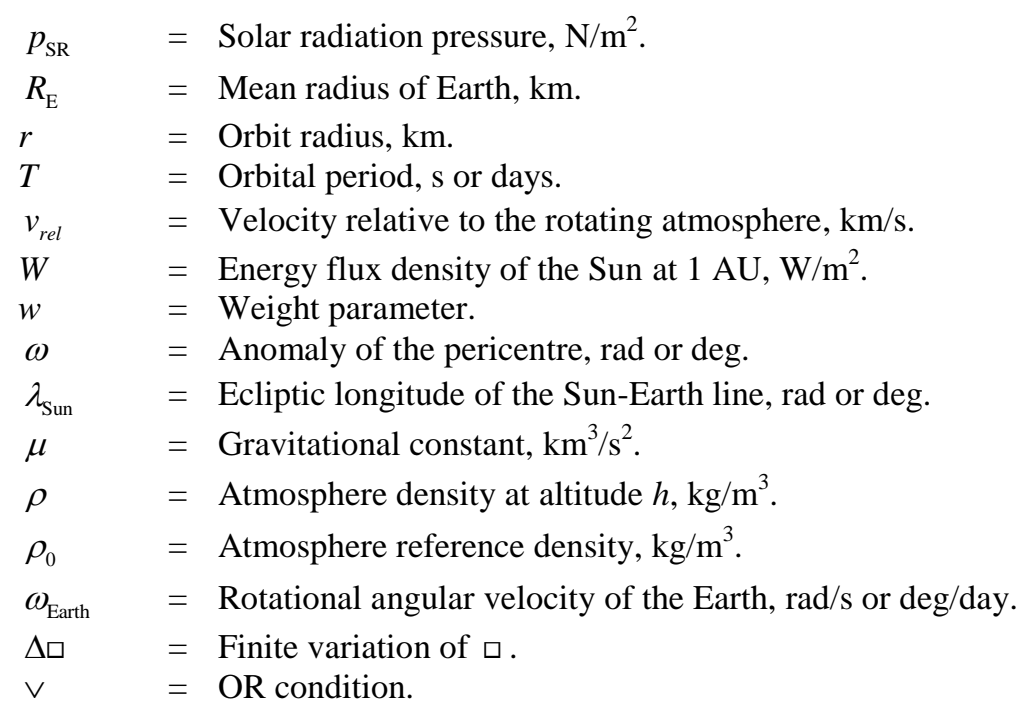

\section{Subscripts}

$a=$ Semi-major axis.

Drag $=$ Due to atmospheric drag.

$e \quad=$ Eccentricity.

Earth = Relative to the Earth.

ecl, enter $=$ Entry into eclipse.

ecl, exit $=$ Exit from eclipse.

$r \quad=$ Component in the radial direction.

$p \quad=$ Relative to the perigee.

Sun $\quad=$ Relative to the Sun.

$\mathrm{SRP}=$ Due to solar radiation pressure.

up $\quad=$ Upper value.

$\theta \quad=$ Component in the transverse direction.

$\omega=$ Anomaly of the pericenter.

$2 \pi \quad=$ Value computed over one revolution of true anomaly.

$* \quad=$ Value at the bifurcation.

\section{Superscripts \\ $\wedge \quad=$ Unit vector. \\ - $\quad=$ Secular term. \\ $T=$ Transposed.}

Acronyms

$\mathrm{AU}=$ Astronomical Unit.

MEMS $=$ MicroElectroMechanical Systems.

RHS $\quad=$ Right-Hand Side.

SRP $=$ Solar Radiation Pressure.

\section{Constants}

$\mathrm{AU}=149597870.7 \mathrm{~km}$.

$c_{\text {light }}=299792.458 \mathrm{~km} / \mathrm{s}$.

$R_{\mathrm{E}} \quad=6378.16 \mathrm{~km}$.

$W \quad=1367 \mathrm{~W} / \mathrm{m}^{2}$.

$\mu_{\text {Earth }}=3.986004461921757 \times 10^{5} \mathrm{~km}^{3} / \mathrm{s}^{2}$.

$\mu_{\text {Sun }}=1.3272448769 \times 10^{11} \mathrm{~km}^{3} / \mathrm{s}^{2}$. 


\section{Introduction}

Advances in miniaturization are enabling the development of micro-scale 'smart dust' devices with sensing, computing and communication capabilities for terrestrial applications [1]. Current concepts for functional devices in space have been designed by exploiting existing capabilities, such as satellite-on-a-chip [2-5]. These developments offer the possibility of fabricating vast numbers of micro-spacecraft for use in swarm applications and with launch at low cost. Significantly smaller MEMS (microelectromechanical systems) devices with sensing, computing, bidirectional communication and micro-power sources are currently in laboratory development for terrestrial applications with a displaced volume of order $10 \mathrm{~mm}^{3}$ [6]. For space applications Atchison and Peck designed a passive, sun-pointing millimeter-scale solar sail, whose bus is constituted by a $1 \mathrm{~cm}$ square and $25 \mu \mathrm{m}$ thick silicon microchip. The proposed design integrates solar cells and communications in a solid state device as a 'SpaceChip' [7].

The deployment of vast numbers of such 'SpaceChips' will enable future missions, such as global sensor networks for Earth observation and communications, distributed space missions for multi-point, real-time sensing for space science (space weather, geomagnetic physics, reflectometry), geo-engineering applications, interplanetary exploration in support of conventional spacecraft, or deployment in the vicinity of a large spacecraft for diagnostic or environmental detection purposes. The considerably smaller dimensions of SpaceChips envisage their deployment in orbit from a CubeSat or as piggy-back on a conventional spacecraft, thus allowing significant launch cost savings [8].

As an early example of a SpaceChip-scale swarm, project West Ford in 1963 placed a ring of $4.8 \times 10^{8}$ copper dipole antennas $(1.78 \mathrm{~cm}$ long needles, with a diameter of $17.8 \mu \mathrm{m})$ into orbit to allow passive global radio communications [9]. The motion of the individual dipoles, from dispensing to final re-entry through the atmosphere was both modeled and observed.

The realization of these new concepts requires an understanding of orbital dynamics at extremes of spacecraft length-scale. The significantly higher area-to-mass ratio of such devices, with respect to conventional spacecraft, requires new insights into orbital dynamics since perturbations such as solar radiation pressure (SRP) and aerodynamic drag can become dominant with respect to the Earth's gravity. Rather than counteracting these disturbances to the natural Keplerian motion, the interaction physics at small length-scales can be exploited and passive methods for orbit design can be envisaged without the use of active orbit control. This paper presents an 
analysis of long-lived orbits for 'smart dust' devices which use the energy input from asymmetric solar radiation pressure to offset the energy dissipation of atmospheric drag. This is of key importance since these small devices have a short lifetime under the effect of air drag alone due to their extremely high area-to-mass ratio.

The effect of natural perturbations on small particles has been studied extensively in the vast literature on the dynamics of cosmic dust in the solar system whose motion is influenced by solar gravity, solar radiation pressure, the Poynting-Robertson drag force, planetary oblateness and electromagnetic forces [10-14]. The effect of solar radiation pressure, zonal and tesseral harmonics of the Earth's gravitational potential, luni-solar third body perturbations and atmospheric drag on high area-to-mass ratio objects are also of particular interest to explain the long-term dynamical evolution of small debris particles released into Earth orbit. For example, observations and high accuracy numerical integrations led to the discovery of a class of objects which remain in orbit for long durations due to the effect of solar radiation pressure in the synchronous and semi-synchronous orbital regime [15, 16]. Furthermore, the effects of solar radiation pressure have been observed since 1960 in the orbital behavior of satellites such as the ECHO balloon [17], Vanguard [18] and many others [19] and are exploited as the main propulsive force for solar sailing, when exerted on a large reflective membrane [20, 21]. For example, McInnes at al. showed that a small solar sail can be used to artificially precess the apse line of an orbit, to provide sun-synchronous tracking of the geomagnetic tail [22]. Oyama at al. extended the analysis to the entire phase-space and defined the Hamiltonian of the system to investigate the evolution of the orbital elements [23]. Due to the characteristics of the orbit selected for the geomagnetic tail exploration mission, only solar radiation pressure was considered and Earth eclipses were neglected. Under these assumptions an equilibrium precessing orbit can be found analytically. De Juan Ovelar et al. explored the possible exploitation of the effects of solar radiation pressure on artificial nano-spheres and the design of a coating for spherical particles to engineer the ratio between the radiation pressure and the gravitational force. Earth-Mars transfer orbits were designed by using the particle coating as design parameter [24].

This paper investigates how the perturbations of solar radiation pressure and atmospheric drag can be balanced on average to obtain long-lived Earth centered orbits for swarms of 'smart dust' devices, without the use of active control. Given the initial orbital elements of the spacecraft, the shadow geometry is determined as a function of semi-major axis, eccentricity and angular displacement between the Sun-Earth line $\lambda_{\text {Sun }}$ and the orbit pericentre $\omega$. The secular change of the in-plane orbital elements over a single orbit revolution is then evaluated analytically. An expression is derived for the variation of Keplerian elements due to solar radiation pressure, which takes into 
account the Earth's shadow through an analytic expression for the exit and entry true anomaly from/into eclipses. An analysis of the change in orbital elements due to solar radiation pressure and atmospheric drag on such small devices is then performed. A search for initial orbital conditions for long-lived orbits is performed through a global search over a wide range of orbit eccentricities, altitudes of the pericentre and values of $\omega-\lambda_{\text {Sun }}$, and using local optimization through non-linear programming. The requirement that the precession of the apse line of the orbit is synchronous with the Sun is imposed in order to ensure the same conditions on the solar radiation pressure perturbation at each revolution. Again, such orbit precession has been investigated for the effect of solar radiation pressure alone without eclipses for solar sail applications [22, 23].

From this initial analysis the paper presents families of long-lived orbits for swarms of 'smart dust' devices. The conditions for long-lived orbits are identified in the orbit element phase-space and numerical integration of the secular variation of orbital elements is used to characterize their long-term evolution. Different families of orbits are presented. In those regions of the phase-space where the effect of atmospheric drag is negligible, equilibrium orbits can be found under the effect of solar radiation only, where the condition of Sun-synchronous apse-line precession is achieved passively, without any propellant mass consumption. If the initial condition is in a certain region around the equilibrium solution set, the long-term evolution follows a librational motion, progressively decaying due to the non-conservative effect of atmospheric drag. Asymmetry in solar radiation pressure due to eclipses leads to modulation of the orbit energy, and families of orbits can be found where the energy gain due to solar radiation pressure balances the energy dissipation due to drag. It is therefore shown that the exploitation of the natural effects of solar radiation pressure and atmospheric drag provides a means of enabling long-lived orbits for future 'smart dust' devices and ensures the final re-entry of the devices so that they do not constitute future space debris.

The paper is organized as follows: Section II introduces the analytical approach to compute the orbit evolution; the initial conditions for long-lived orbits and the method adopted to identify them are described in Section III. Sections IV and V present the solution for long-lived orbits and their long-term evolution. Finally some mission applications for swarms of 'smart dust' devices are proposed in Section VI.

\section{Orbit evolution}

A semi-analytical theory is used to compute the orbit evolution of the spacecraft under the influence of solar radiation pressure and drag. The secular variation of the orbital elements is obtained by averaging Gauss' planetary 
equations in the true anomaly or eccentric anomaly form. As a first approach to explore orbital dynamics at small length-scales, we consider only solar radiation pressure and atmospheric drag, whose perturbing accelerations are proportional to the area-to-mass ratio of the spacecraft, hence their effect is greater for small devices since area-tomass ratio is a strong function of spacecraft length-scale. The model considers a spherical Earth to allow the interaction of energy gain due to SRP and energy dissipation due to atmospheric drag to be clearly investigated; future work will include the influence of the Earth's oblateness, which causes a secular drift of the perigee and the argument of the ascending node. Again, to begin to explore conditions for long-lived orbits we constrain the present study to orbits lying in the ecliptic plane. In the remainder of this section, the semi-analytical model used for orbit propagation will be presented.

\section{A. Secular variation of Keplerian elements due to solar radiation pressure}

We consider a spacecraft on an Earth-centered orbit lying in the ecliptic plane, with the geometry represented in Fig. 1. The satellite is subjected to an acceleration due to solar radiation pressure given by

$$
\begin{aligned}
& a_{r, \mathrm{SRP}}=a_{\mathrm{SRP}} \cos \left(\omega-\lambda_{\mathrm{Sun}}+f\right) \\
& a_{\theta, \mathrm{SRP}}=-a_{\mathrm{SRP}} \sin \left(\omega-\lambda_{\mathrm{Sun}}+f\right)
\end{aligned}
$$

where $f$ is the true anomaly, $a_{r}$ and $a_{\theta}$ are the components of the acceleration $\mathbf{a}_{\mathrm{SRP}}=\left[\begin{array}{ll}a_{r, \mathrm{SRP}} & a_{\theta, \mathrm{SRP}}\end{array}\right]^{T}$ in the radial and transversal directions in the orbital plane, and the characteristic acceleration

$$
a_{\mathrm{SRP}}=\frac{p_{\mathrm{SR}} c_{R} A_{\odot}}{m}
$$

where $p_{\mathrm{SR}}$ is the solar pressure $p_{\mathrm{SR}}=\frac{W}{c_{\text {light }}}=4.56 \times 10^{-6} \frac{\mathrm{N}}{\mathrm{m}^{2}}$ (with $W$ the energy flux density of the Sun at $1 \mathrm{AU}$ and $c_{\text {light }}$ the speed of light), $c_{\mathrm{R}}$ the reflectivity coefficient that measures the momentum exchange between incoming radiation and the spacecraft [25], $A_{\odot}$ is the area exposed to the Sun, which is considered constant in the following analysis and $m$ is the mass of the satellite. The angle $\omega$ is the argument of the orbit perigee and $\lambda_{\text {sun }}$ describes the direction of the Sun-line. Both $\omega$ and $\lambda_{\text {Sun }}$ are measured with respect to a fixed arbitrary direction, such that the angle $\omega-\lambda_{\text {Sun }}$ represents the angular displacement between the orbit pericentre and the Sun-line direction. 


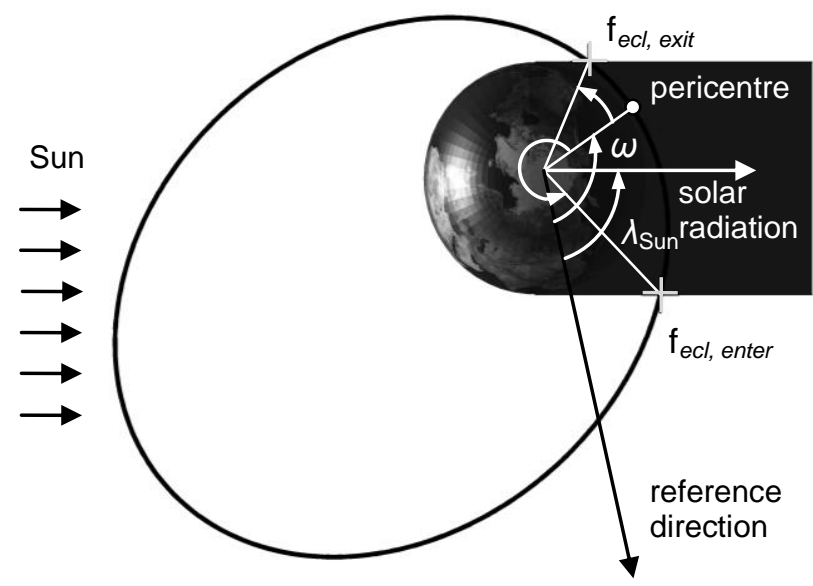

Fig. 1 Orbit geometry.

To investigate the effect of SRP on the spacecraft orbit, we consider the planar terms of the Gauss equations, written as function of the true anomaly $f[26]$

$$
\begin{aligned}
& \frac{d a}{d f}=\frac{2 p r^{2}}{\mu_{\text {Earth }}\left(1-e^{2}\right)^{2}}\left(e \sin f \cdot a_{r}+\frac{p}{r} \cdot a_{\theta}\right) \\
& \frac{d e}{d f}=\frac{r^{2}}{\mu_{\text {Earth }}}\left(\sin f \cdot a_{r}+\left(1+\frac{r}{p}\right) \cos f \cdot a_{\theta}+e \frac{r}{p} \cdot a_{\theta}\right) \\
& \frac{d \omega}{d f}=\frac{r^{2}}{\mu_{\text {Earth }} e}\left(-\cos f \cdot a_{r}+\left(1+\frac{r}{p}\right) \sin f \cdot a_{\theta}\right)
\end{aligned}
$$

where $\mu_{\text {Earth }}$ is the gravitational constant of the Earth, $a, e$ and $\omega$ are the in-plane orbital elements, $r$ is the orbit radius, and $p$ the semilatus rectum. An averaging technique is used to compute the long-term variation of the orbital elements, i.e. Eqs. (3) are integrated in true anomaly, considering the other orbital elements to be constant over one orbit revolution. In the case of a constant disturbing acceleration in a fixed inertial direction defined in Eq. (1), Eqs. (3) can be solved in closed form to find the primitive functions $\mathrm{f}_{a}, \mathrm{f}_{e}, \mathrm{f}_{\omega}$ for semi-major axis, eccentricity and anomaly of the pericenter:

$$
\begin{aligned}
& \mathrm{f}_{a}\left(a, e, \omega-\lambda_{\text {Sun }}\right)=\int \frac{d a}{d f} d f+c_{a} \\
& \mathrm{f}_{e}\left(a, e, \omega-\lambda_{\text {Sun }}\right)=\int \frac{d e}{d f} d f+c_{e} \\
& \mathrm{f}_{\omega}\left(a, e, \omega-\lambda_{\text {Sun }}\right)=\int \frac{d \omega}{d f} d f+c_{\omega}
\end{aligned}
$$


Integration constants $c_{a}, c_{e}$ and $c_{\omega}$ are introduced in the primitive functions but they are removed when the primitive function is evaluated at two limits of integration. Substituting Eq. (1) in Eqs. (3) the indefinite integrals can be computed, as shown in Eqs. (4). After some algebraic manipulations we obtain:

$$
\begin{aligned}
& \mathrm{f}_{a}=-\frac{2 a^{3}\left(1-e^{2}\right)}{\mu_{\text {Earth }}} a_{\mathrm{SRP}}\left(\frac{\cos \left(\omega-\lambda_{\mathrm{Sun}}\right)+e \sin \left(\omega-\lambda_{\mathrm{Sun}}\right) \sin f}{e(1+e \cos f)}\right)+c_{a} \\
& \mathrm{f}_{e}=\frac{a^{2}\left(1-e^{2}\right)^{2}}{\mu_{\text {Earth }}} a_{\mathrm{SRP}}\left(\sin \left(\omega-\lambda_{\mathrm{Sun}}\right)\left(-\frac{3}{2} \frac{E}{\left(1-e^{2}\right)^{3 / 2}}+\frac{\sin f\left(\cos f\left(-8 e^{4}+10 e^{2}-2\right)+6 e\left(1-e^{2}\right)\right)}{4\left(1-e^{2}\right)^{2}(1+e \cos f)^{2}}\right)+\right. \\
& \left.-\frac{\cos \left(\omega-\lambda_{\text {Sun }}\right)}{2 e^{2}(1+e \cos f)^{2}}\left(1+2 e \cos f+e^{2}\right)\right)+c_{e} \\
& \mathrm{f}_{\omega}=-\frac{a^{2}\left(1-e^{2}\right)^{2}}{\mu_{\text {Earth }} e} a_{\mathrm{SRP}}\left(\cos \left(\omega-\lambda_{\mathrm{Sun}}\right)\left(\frac{3}{2} \frac{E}{\left(1-e^{2}\right)^{3 / 2}}-\frac{e \sin f}{\left(1-e^{2}\right)(1+e \cos f)}-\frac{1}{2} \frac{(e+\cos f) \sin f}{\left(1-e^{2}\right)(1+e \cos f)^{2}}\right)+\right. \\
& \left.+\sin \left(\omega-\lambda_{\text {Sun }}\right) \frac{1+2 e \cos f}{2 e^{2}(1+e \cos f)^{2}}\right)+c_{\omega}
\end{aligned}
$$

where $E$ is the eccentric anomaly which is function of the true anomaly. Note that Eqs. (5) have been obtained under the assumption that the orbit lies in the ecliptic plane. Eqs. (5) assume that the disturbing acceleration $a_{\mathrm{SRP}}$ is constant when the spacecraft is in sunlight, i.e., the variation of the solar flux over time is neglected, and the exposed area $A_{\odot}$ in Eq. (2) is considered constant (i.e., the spacecraft has a spherical shape or the attitude of the spacecraft is kept constant with respect to the Sun-line). At this point, the total variation of the orbital elements can be evaluated over the orbit arc in which the spacecraft is in sunlight: $\left[0, f_{\text {ecl,enter }}\right],\left[f_{\text {ecl, exit }}, 2 \pi\right]$, which is function of $a, e$, $\omega-\lambda_{\text {Sun }}$ at the orbit pericentre and the true anomaly at which the satellite enters and exits the eclipses, $f_{\text {ecl,enter }}$ and $f_{\text {ecl, exit }}$. Since we are assuming the orbital elements and $a_{\mathrm{SRP}}$ to be fixed over one orbit revolution, at their value at the pericentre, the total variation of the orbital elements can be written as:

$$
\begin{aligned}
& \Delta a_{\mathrm{SRP}, 2 \pi}\left(a, e, \omega-\lambda_{\mathrm{Sun}}, f_{\mathrm{ecl}, \text { exit }}, f_{\text {ecl, enter }}\right)=\left[\mathrm{f}_{a}\right]_{0}^{f_{\mathrm{ecl}, \text { enter }}}+\left[\mathrm{f}_{a}\right]_{f_{\text {ecl, exit }}}^{2 \pi}=\left[\mathrm{f}_{a}\right]_{f_{\text {ecl, exit }}}^{f_{\text {ecl enter }}} \\
& \Delta e_{\mathrm{SRP}, 2 \pi}\left(a, e, \omega-\lambda_{\text {Sun }}, f_{\text {ecl, exit }}, f_{\text {ecl, enter }}\right)=\left[\mathrm{f}_{e}\right]_{0}^{f_{\text {ecl, enter }}}+\left[\mathrm{f}_{e}\right]_{f_{\text {ecl, exit }}^{2 \pi}}^{2 \pi}=\left[\mathrm{f}_{e}\right]_{f_{\text {ecl, exit }}}^{f_{\text {ecter }}} \\
& \Delta \omega_{\mathrm{SRP}, 2 \pi}\left(a, e, \omega-\lambda_{\text {Sun }}, f_{\text {ecl, exit }}, f_{\text {ecl, enter }}\right)=\left[\mathrm{f}_{\omega}\right]_{0}^{f_{\text {ecl, enter }}}+\left[\mathrm{f}_{\omega}\right]_{f_{\text {ecl, exit }}}^{2 \pi}=\left[\mathrm{f}_{\omega}\right]_{f_{\text {ecl, exit }}}^{f_{\text {ecl enter }}}
\end{aligned}
$$


In the planar case we consider (i.e., the orbit lies in the ecliptic plane), the arguments of true anomaly when the spacecraft enters and leaves the Earth's shadow can be expressed as a closed-form function of the orbital elements: $f_{\text {ecl, enter }}\left(a, e, \omega-\lambda_{\text {Sun }}\right), f_{\text {ecl, exit }}\left(a, e, \omega-\lambda_{\text {Sun }}\right)$. Assuming that the parallax of the Sun is negligible, the arguments of true anomaly at which the satellite enters and exits eclipses are given by the following systems (see Fig. 1):

$$
\begin{array}{ll}
f_{\text {ecl, exit }}\left(a, e, \omega-\lambda_{\text {Sun }}\right): & \left\{\begin{array}{l}
r \cos \left(\omega-\lambda_{\text {Sun }}+f\right)>0 \\
r \sin \left(\omega-\lambda_{\text {Sun }}+f\right)=+R_{\mathrm{E}}
\end{array}\right. \\
f_{\text {ecl, enter }}\left(a, e, \omega-\lambda_{\text {Sun }}\right): & \left\{\begin{array}{l}
r \cos \left(\omega-\lambda_{\text {Sun }}+f\right)>0 \\
r \sin \left(\omega-\lambda_{\text {Sun }}+f\right)=-R_{\mathrm{E}}
\end{array}\right.
\end{array}
$$

where $R_{\mathrm{E}}$ is the mean radius of the Earth, and $r$ the orbital radius. The expressions for $f_{\text {ecl, exit }}\left(a, e, \omega-\lambda_{\text {Sun }}\right)$ and $f_{\text {ecl, enter }}\left(a, e, \omega-\lambda_{\text {Sun }}\right)$ can be found analytically, after some algebraic manipulation and are given by

$$
\begin{aligned}
& f_{\text {ecl, exit }}=\mp \arccos \left[\frac{-R_{\mathrm{E}} d_{\text {exit }}+a\left(1-e^{2}\right) \cos \left(\omega-\lambda_{\text {Sun }}\right) \sqrt{\left(1-e^{2}\right)\left(a^{2}\left(1-e^{2}\right)-R_{\mathrm{E}}^{2}-2 a e R_{\mathrm{E}} \sin \left(\omega-\lambda_{\text {Sun }}\right)\right)}}{a^{2}\left(1-e^{2}\right)^{2}+e^{2} R_{\mathrm{E}}^{2}-2 a e R_{\mathrm{E}}\left(1-e^{2}\right) \sin \left(\omega-\lambda_{\text {Sun }}\right)}\right] \\
& f_{\text {ecl, enter }}=\mp \arccos \left[\frac{-R_{\mathrm{E}} d_{\text {enter }}+a\left(1-e^{2}\right) \cos \left(\omega-\lambda_{\text {Sun }}\right) \sqrt{\left(1-e^{2}\right)\left(a^{2}\left(1-e^{2}\right)-R_{\mathrm{E}}^{2}+2 a e R_{\mathrm{E}} \sin \left(\omega-\lambda_{\text {Sun }}\right)\right)}}{a^{2}\left(1-e^{2}\right)^{2}+e^{2} R_{\mathrm{E}}^{2}+2 a e R_{\mathrm{E}}\left(1-e^{2}\right) \sin \left(\omega-\lambda_{\text {Sun }}\right)}\right]
\end{aligned}
$$

where we denote

$$
\begin{aligned}
& d_{\text {exit }}=e R_{\mathrm{E}}-a\left(1-e^{2}\right) \sin \left(\omega-\lambda_{\text {Sun }}\right) \\
& d_{\text {enter }}=e R_{\mathrm{E}}+a\left(1-e^{2}\right) \sin \left(\omega-\lambda_{\text {Sun }}\right)
\end{aligned}
$$

Equations (8) are valid also in the circular case, $e=0$, if an arbitrary value of the anomaly of the pericentre $\omega$ is chosen. The $\mp$ sign in Eqs. (8) identifies the two symmetric positions which straddle the pericentre (and the line of apsides) as shown in Fig. 2. The "o" symbol indicates the two possible solutions of Eq. (8a) for $f_{\text {ecl,exit }}\left(f_{\text {ecl, exit }}^{-}\right.$and $f_{\text {ecl,exit }}^{+}$, whereas the " $\diamond$ " symbol indicates the two possible solutions of Eq. (8b) for $f_{\text {ecl, enter }}\left(f_{\text {ecl, enter }}^{-}\right.$and $f_{\text {ecl,enter }}^{+}$). The superscript "+" indicates the solution with an angle between 0 and $\pi$ and the superscript "-" indicates the solution with an angle between $\pi$ and $2 \pi$. An algorithm was developed to identify the correct solution of each of 
Eqs. (8) corresponding to the actual shadow boundary, by verifying whether the orbit pericentre or apocentre is in shadow.

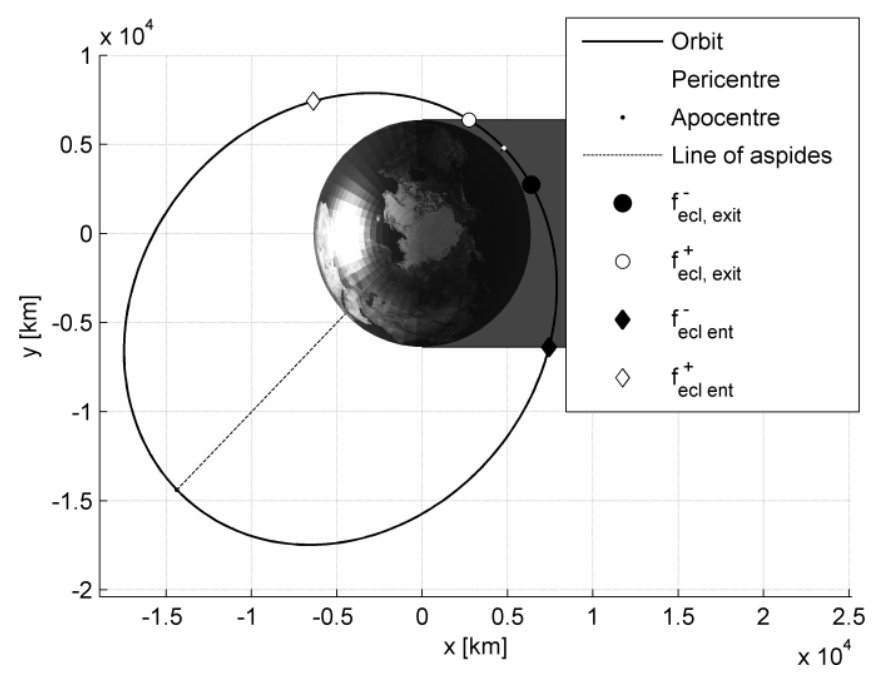

Fig. 2 Eclipse geometry: solutions of Eqs. (8). The "o" symbol indicates the two possible solutions for the equation of the exit true anomaly; the " $\diamond$ " symbol indicates the two possible solutions for the equation of the entry true anomaly. The superscript " + " indicates the solution with an angle between 0 and $\pi$, the superscript "_" indicates the solution with an angle between 0 and $2 \pi$.

The expressions for $f_{\text {ecl, exit }}\left(a, e, \omega-\lambda_{\text {Sun }}\right)$ and $f_{\text {ecl, enter }}\left(a, e, \omega-\lambda_{\text {Sun }}\right)$ given by Eqs. (8) can be substituted into Eqs. (6) to provide the actual variation of orbital elements considering the Earth's shadow. If the spacecraft does not enter into eclipse, $f_{\text {ecl, enter }}=f_{\text {ecl, exit }}+2 \pi$, some terms of Eqs. (6) vanish (the terms containing $\sin f$ and cos $f$ ), in particular the variation of semi-major axis goes to zero. In the case of no eclipse, Eqs. (6) simplify to the formulation used by McInnes et al. [22] and Oyama et al. [23].

The secular and long-period rate of change of the orbital elements can be obtained by dividing Eqs. (6) by the Keplerian orbital period

$$
T=2 \pi \sqrt{\frac{a^{3}}{\mu_{\text {Earth }}}}
$$

thus giving

$$
\frac{d \bar{a}}{d t}_{\mathrm{SRP}}=\frac{\Delta a_{\mathrm{SRP}, 2 \pi}}{2 \pi} \sqrt{\frac{\mu_{\mathrm{Earth}}}{a^{3}}}
$$




$$
\begin{gathered}
\frac{d \bar{e}}{d t}_{\mathrm{SRP}}=\frac{\Delta e_{\mathrm{SRP}, 2 \pi}}{2 \pi} \sqrt{\frac{\mu_{\mathrm{Earth}}}{a^{3}}} \\
\frac{d \bar{\omega}}{d t}_{\mathrm{SRP}}=\frac{\Delta \omega_{\mathrm{SRP}, 2 \pi}}{2 \pi} \sqrt{\frac{\mu_{\mathrm{Earth}}}{a^{3}}}
\end{gathered}
$$

where the overbar is used to indicate the secular variation. The line of apsides of the ellipse will rotate due to the perturbing solar radiation pressure acceleration, with a mean rate of precession given by the Eq. (11c) that can be rewritten to express the perigee precession with respect to the Sun-line, introducing the rotational rate of the Earth around the Sun $n_{\text {Earth-Sun }}$.

$$
{\frac{d\left(\bar{\omega}-\bar{\lambda}_{\mathrm{Sun}}\right)}{d t} \quad \mathrm{SRP}}_{2 \pi}=\frac{\Delta \omega_{\mathrm{SRP}, 2 \pi}}{\frac{\mu_{\text {Earth }}}{a^{3}}}-n_{\text {Earth-Sun }}
$$

\section{B. Secular variation of Keplerian elements due to atmospheric drag}

For spacecraft orbits with a low perigee the motion is also influenced by atmospheric drag acceleration

$$
\mathbf{a}_{\text {Drag }}=-\frac{1}{2} \frac{c_{D} A_{\text {Drag }}}{m} \rho v_{r e l}^{2} \hat{\mathbf{v}}_{\text {rel }}
$$

where $c_{D}$ is the drag coefficient, $A_{\text {Drag }}$ is the effective cross-sectional area of the spacecraft and $m$ its mass, $v_{r e l}$ is the velocity relative to the rotating atmosphere and $\hat{\mathbf{v}}_{\text {rel }}$ the corresponding unit vector. The secular disturbing effect on the orbit due to atmospheric drag can be modeled analogously to the case of solar radiation pressure. Starting from the Gauss equations written as function of the eccentric anomaly, King-Hele [27] derived equations that express the secular perturbation on the orbital elements due to atmospheric drag. These equations are based on the assumption of a time-independent, spherically-symmetric atmosphere with a density that varies exponentially with altitude $h$, according to

$$
\rho=\rho_{0} \exp \left[-\frac{h-h_{0}}{H}\right]
$$

where $\rho_{0}$ is the reference density at the reference altitude $h_{0}$ and $H$ is the scale height, whose values are taken from tables [25]. If we neglect atmospheric rotation (i.e., the angular velocity of the Earth $\omega_{\text {Earth }}$ is zero), the variation of 
$\Delta \omega_{\text {Drag, } 2 \pi}$ due to drag is zero, while the change of the in-plane orbital elements over a single revolution is given by [28]:

$$
\begin{aligned}
& \Delta e_{\operatorname{Drag}, 2 \pi}=-2 \pi \delta a \rho_{p} \exp [-c]\left\{I_{1}+\frac{e}{2}\left(I_{0}+I_{2}\right)-\frac{e^{2}}{8}\left(5 I_{1}-I_{3}\right)-\frac{e^{3}}{16}\left(5 I_{0}+4 I_{2}-I_{4}\right)+O\left(e^{4}\right)\right\} \\
& \Delta h_{p \operatorname{Drag}, 2 \pi}=-2 \pi \delta a^{2} \rho_{p} \exp [-c]\left\{\left(I_{0}-I_{1}\right)-\frac{e}{2}\left(3 I_{0}-4 I_{1}+I_{2}\right)+\frac{e^{2}}{8}\left(6 I_{0}-11 I_{1}+6 I_{2}-I_{3}\right)+\right. \\
& \left.-\frac{e^{3}}{16}\left(7 I_{0}-12 I_{1}+8 I_{2}-4 I_{3}+I_{4}\right)+O\left(e^{4}\right)\right\} \\
& \Delta a_{\text {Drag, } 2 \pi}=\frac{\Delta h_{p \text { Drag, } 2 \pi}+a \cdot \Delta e_{\text {Drag, } 2 \pi}}{1-e} \\
& \Delta \omega_{\text {Drag, } 2 \pi}=0
\end{aligned}
$$

where $\rho_{p}$ is the density at the orbit perigee, computed through Eq. (13), the factor $c=\frac{a e}{H}, I_{k}$ are the modified Bessel functions of the first kind of order $k$ and argument $c$ [29], and $\delta=\frac{Q A_{\mathrm{drag}} c_{D}}{m}$ (where the drag coefficient $c_{D}$ is considered constant, and the factor $Q$ is equal to 1 for static atmosphere). Note that Eqs. (14) are valid up to the order of eccentricity indicated, within the range $0.01 \leq e \leq 0.8$. Blitzer provides an expression of both $\Delta a_{\operatorname{Drag}, 2 \pi}$, $\Delta e_{\text {Drag, } 2 \pi}$, and $\Delta h_{p \text { Drag, } 2 \pi}$, which are interdependent [28]. We compute $\Delta e_{\text {Drag, } 2 \pi}$ and $\Delta h_{p \text { Drag, } 2 \pi}$ and then derive $\Delta a_{\text {Drag, } 2 \pi}$ from those two expressions as shown in Eqs. (14). This choice minimizes the numerical errors when the equations giving the corresponding rate of change (see Eqs. (15) in the following) are integrated over a long duration, because smaller errors (due to the higher terms in eccentricity) are accumulated in the computation of the perigee altitude, which determines the value of the air density.

Analogously to Eqs. (11), we obtain the secular and long-period rate of change of the orbital elements by dividing Eqs. (14) by the Keplerian orbital period:

$$
\begin{aligned}
& \frac{d \bar{a}}{d t}{ }_{\text {Drag }}=\frac{\Delta a_{\text {Drag, } 2 \pi}}{2 \pi} \sqrt{\frac{\mu_{\text {Earth }}}{a^{3}}} \\
& \frac{d \bar{e}}{d t_{\text {Drag }}}=\frac{\Delta e_{\text {Drag, } 2 \pi}}{2 \pi} \sqrt{\frac{\mu_{\text {Earth }}}{a^{3}}} \\
& \frac{d \bar{\omega}}{d t_{\text {Drag }}}=0
\end{aligned}
$$

The total secular variation of the orbital elements can then be expressed as 


$$
\begin{aligned}
& \frac{d \bar{a}}{d t}=\frac{d \bar{a}}{d t_{\mathrm{SRP}}}+\frac{d \bar{a}}{d t}{ }_{\mathrm{Drag}} \\
& \frac{d \bar{e}}{d t}=\frac{d \bar{e}}{d t_{\mathrm{SRP}}}+\frac{d \bar{e}}{d t_{\mathrm{Drag}}} \\
& \frac{d \bar{\omega}}{d t}=\frac{d \bar{\omega}}{d t_{\mathrm{SRP}}}
\end{aligned}
$$

The accuracy of Eqs. (6), Eqs. (14) and Eqs. (16) was verified in [30] by comparison with the numerical integration of the dynamics in Cartesian coordinates, using the expression for the disturbing accelerations Eq. (1) and Eq. (12).

\section{Conditions for long-lived orbits}

To study the effect on the satellite's orbit of the influence of both solar radiation pressure and atmospheric drag, the secular variation of the Keplerian elements over a single orbital revolution can be computed by adding Eqs. (6) to Eqs. (14), according to the principle of superposition. This can be done under the assumption that the coupling between SRP and atmospheric drag is negligible to first order. This simplifying assumption was verified through numerical integration in Ref. [30]. Therefore, we obtain:

$$
\begin{aligned}
& \Delta a_{\text {Drag }+ \text { SRP }, 2 \pi}=\Delta a_{\text {Drag, } 2 \pi}+\Delta a_{\mathrm{SRP}, 2 \pi} \\
& \Delta e_{\text {Drag }+ \text { SRP }, 2 \pi}=\Delta e_{\text {Drag, } 2 \pi}+\Delta e_{\mathrm{SRP}, 2 \pi} \\
& \Delta \omega_{\text {Drag }+\mathrm{SRP}, 2 \pi}=\Delta \omega_{\text {Drag, } 2 \pi}+\Delta \omega_{\mathrm{SRP}, 2 \pi}
\end{aligned}
$$

The search for equilibrium orbits imposes three conditions to be satisfied. The total variation of semi-major axis and eccentricity due to SPR and drag must be zero, i.e., the combined effect of the two natural perturbations must cancel. Moreover, the Sun-synchronous condition, to maintain the eclipse geometry, imposes the requirement that the change in argument of perigee over one orbit due to SRP (recall from Eqs. (14) that $\Delta \omega_{\text {Drag, } 2 \pi}=0$ ) must be equal to the angular displacement of the Earth around the Sun (i.e., the apparent rotation of the Sun around an Earth inertial system) over one orbital period of the spacecraft, such that the net change of $\omega-\lambda_{\text {Sun }}$ is zero.

$$
\left\{\begin{array}{l}
\Delta a_{\mathrm{SRP}, 2 \pi}+\Delta a_{\mathrm{Drag}, 2 \pi}=0 \\
\Delta e_{\mathrm{SRP}, 2 \pi}+\Delta e_{\mathrm{Drag}, 2 \pi}=0 \\
\Delta \omega_{\mathrm{SRP}, 2 \pi}=\Delta \lambda_{\mathrm{Sun}, 2 \pi}
\end{array}\right.
$$


with $\Delta \lambda_{\text {Sun, } 2 \pi}=n_{\text {Earth-Sun }} \cdot T(a), T$ being the orbital period, given by Eq. (10), which depends only on the semi-major axis $a$. Note that the third equation of system Eq. (17) in the simplified case without Earth shadow was adopted by McInnes et al. [22] and Oyama et al. [23] for determining the required characteristic acceleration of a solar sail to provide Sun-synchronous apse-line precession.

Before focusing on the solution of Eqs. (17), we analyze the behavior of $\Delta a_{\mathrm{SRP}, 2 \pi}, \Delta a_{\mathrm{Drag}, 2 \pi}, \Delta e_{\mathrm{SRP}, 2 \pi}$, $\Delta e_{\text {Drag, } 2 \pi}$, and $\Delta \omega_{\mathrm{SRP}, 2 \pi}$ as a function of the initial conditions of the spacecraft (i.e., the values of the Keplerian elements $a, e$, and $\omega-\lambda_{\text {Sun }}$ at the orbit pericentre). Fig. 3, Fig. 4 and Fig. 6 show respectively the variation of semimajor axis, eccentricity and anomaly of the pericentre over a single orbit revolution due to SRP and drag, as a function of the initial condition in eccentricity and angular displacement with respect to the Sun-direction $\omega-\lambda_{\text {Sun }}$. The characteristic area-to-mass ratio for this example is reported in Table 1 (SpaceChip 2), however the following analysis is valid for all device length-scales proposed in Section IV.A. Due to the asymmetry introduced by eclipses, the net change in semi-major axis due to solar radiation pressure is not zero (apart for $\omega-\lambda_{\text {Sun }}=0$ or $\pi$ ) and its sign depends on the orientation of the orbit with respect to the Sun-line (see Fig. 3a). Due to the presence of the Earth's shadow, the increase in energy that the spacecraft gains over the half of the orbit while moving away from the Sun is not balanced by the energy loss when moving towards the Sun. This effect is greater the more marked is the asymmetry of the orbit geometry with respect to the Sun-line (i.e., $\omega-\lambda_{\text {Sun }}$ far from 0 or $\pi$ ). The effect of drag, instead, is not conservative and causes a continuous energy loss (see Fig. 3b). It is straightforward to see that, when the two effects are superimposed, the zero of $\Delta a_{\mathrm{SRP}, 2 \pi}+\Delta a_{\text {Drag, } 2 \pi}$ moves to values of $\omega-\lambda_{\mathrm{Sun}}$ within the range $0<\omega-\lambda_{\text {Sun }}<\pi$; this can be seen from Fig. 5a. The change of eccentricity over a single orbit under the influence of solar radiation pressure is a function of $\sin \left(\omega-\lambda_{\text {Sun }}\right)$ in addition to other terms due to the presence of eclipses and is equal to zero for $\omega-\lambda_{\text {Sun }}=0$ or $\pi$ (see Fig. 4a). Atmospheric drag causes a constant decrease in eccentricity (see Fig. $4 \mathrm{~b}$ ), hence the superposition of the effects moves the zero of $\Delta e_{\mathrm{SRP}, 2 \pi}+\Delta e_{\mathrm{Drag}, 2 \pi}$ towards a range of angular displacements $\pi<\omega-\lambda_{\text {Sun }}<2 \pi$, as represented in Fig. 5b. Finally the change in anomaly of the pericentre due to solar radiation pressure $\Delta \omega_{\mathrm{SRP}, 2 \pi}$ varies as $\cos \left(\omega-\lambda_{\text {Sun }}\right)$ in addition to other terms due to eclipses (see dark surface in Fig. 6) and it has to counteract the angular rotation of the Earth around the Sun over one orbital period of the 
spacecraft $\Delta \lambda_{\text {Sun, } 2 \pi}$, which is always positive (see light surface in Fig. 6). From Fig. 6 we can conclude that this can be verified for a range of angular displacements $\frac{\pi}{2}<\omega-\lambda_{\text {Sun }}<\frac{3}{2} \pi$.
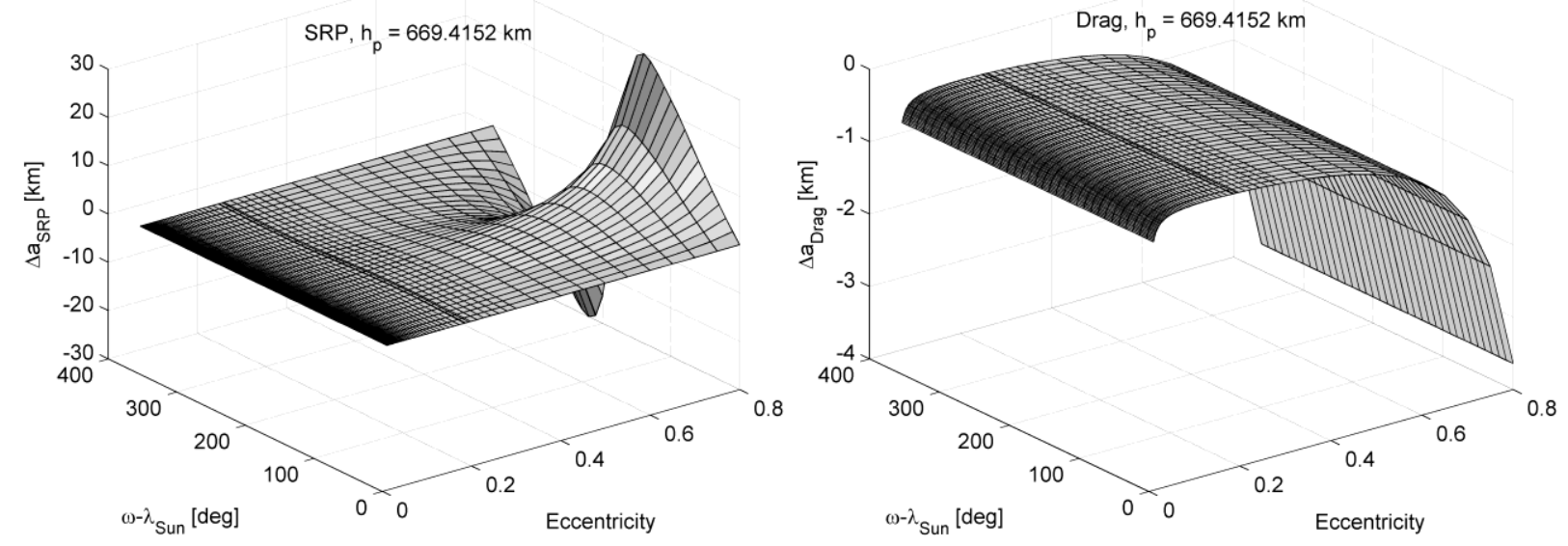

a)

b)

Fig. 3 Variation of semi-major axis over a single orbit revolution for SpaceChip 2 due to SRP and drag as a function of the initial condition in eccentricity and $\omega-\lambda_{\text {Sun }}$ and for a selected value of perigee altitude. a) Change due to SRP, b) change due to atmospheric drag.

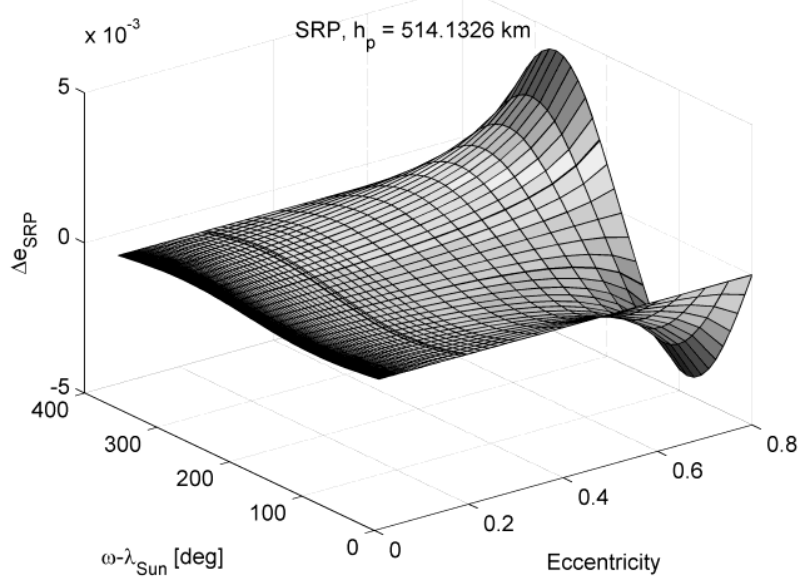

a)

Fig. 4 Variation of eccentricity over a single orbit revolution for SpaceChip 2 due to SRP and drag as a function of the initial conditions in eccentricity and $\omega-\lambda_{\text {Sun }}$ and for a selected value of perigee altitude. a) Change due to SRP, b) change due to atmospheric drag.

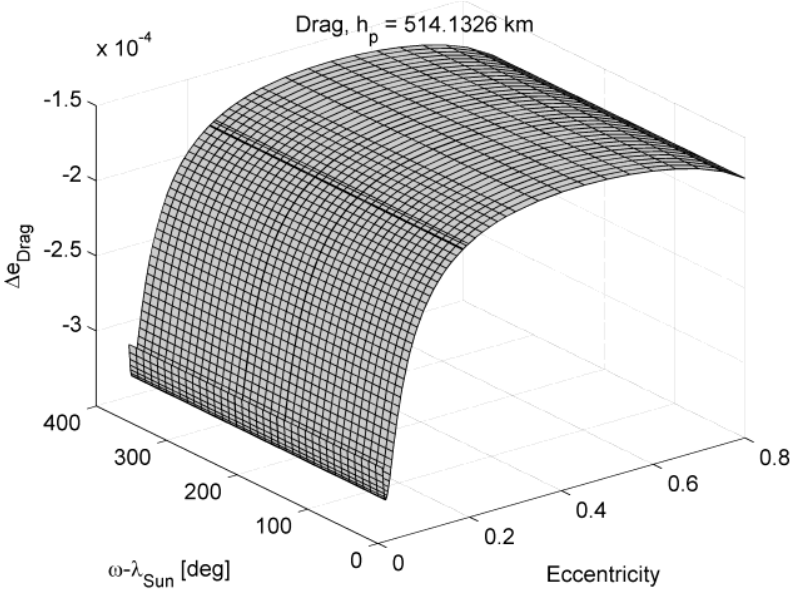

b) 

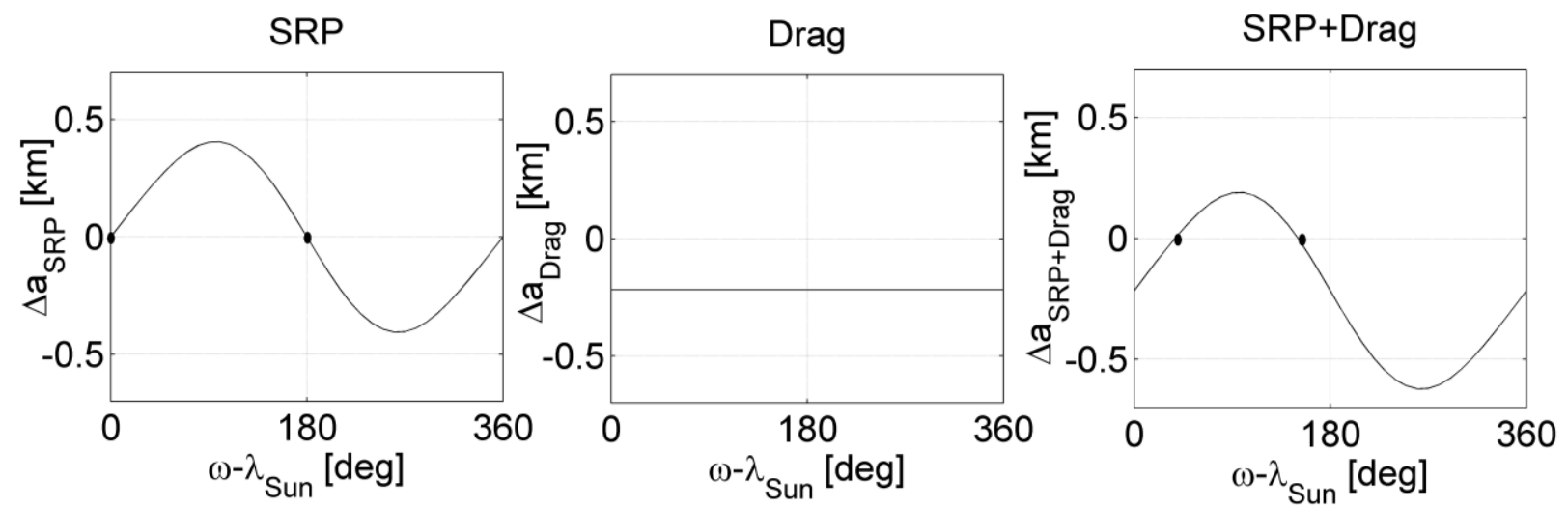

a)
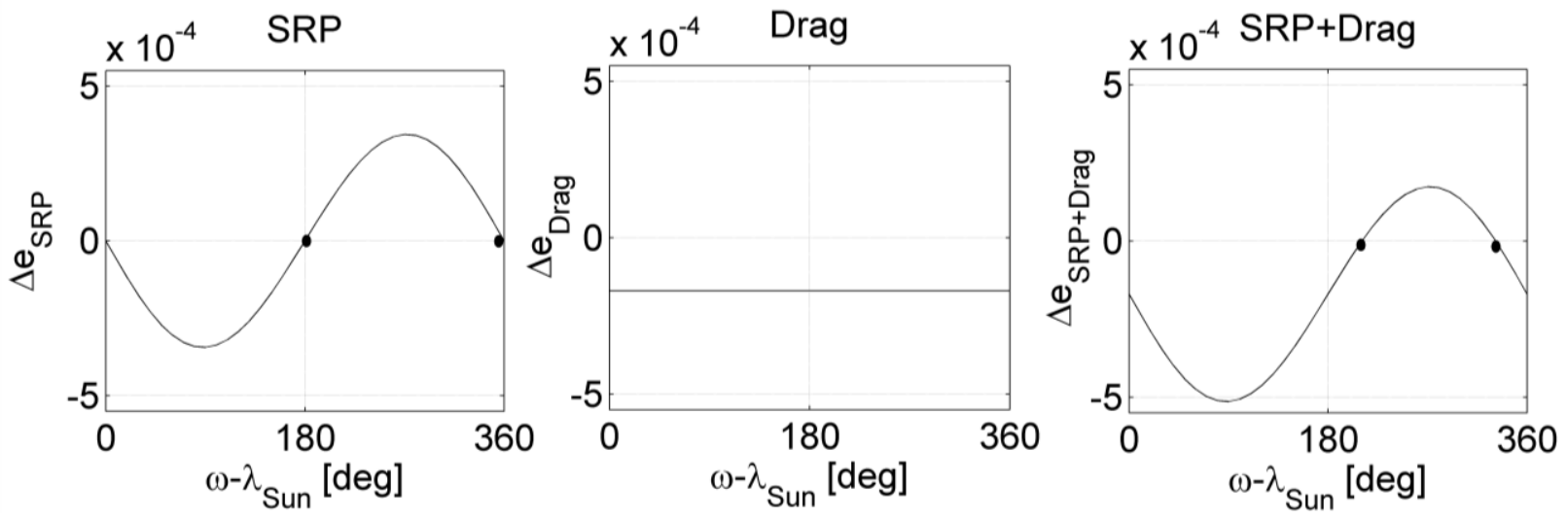

b)

Fig. 5 Variation of orbital elements over a single orbit revolution for SpaceChip 2 as a function of the initial conditions in $\omega-\lambda_{\text {Sun }}$ under the influence of SRP (first column), drag (second column) and SRP + drag (third column). a) Variation of semi-major axis (for a selected value of $h_{p}=669.4152 \mathrm{~km}$ and $\mathrm{e}=\mathbf{0 . 1 9 3 3 5}$ ), and b) variation of semi-major axis (for a selected value of $h_{p}=514.1326 \mathrm{~km}$ and $\mathrm{e}=0.18621$ ).

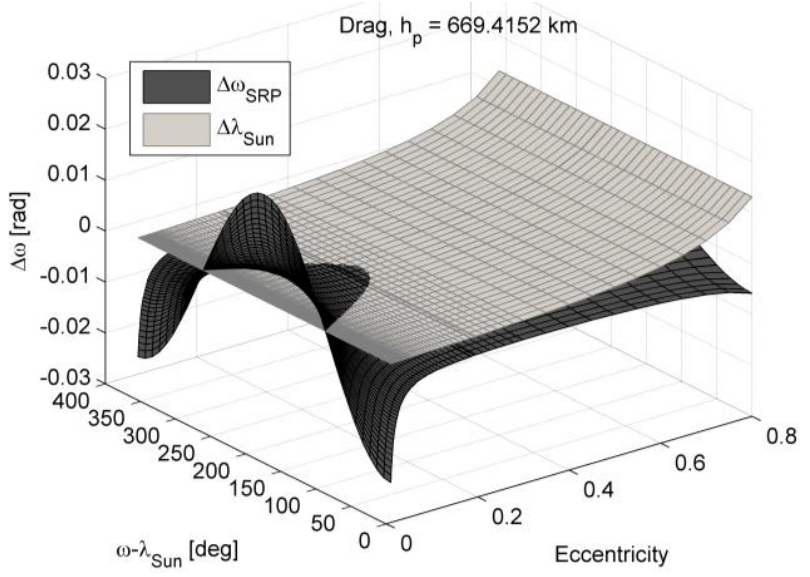

Fig. 6 Intersection between the variation of anomaly of the pericentre due to SRP and precession of the SunEarth line over a single orbit revolution for SpaceChip 2 as a function of the initial condition in eccentricity and $\omega-\lambda_{\text {Sun }}$ and for a selected value of perigee altitude. 
To summarize, from the analysis of Fig. 3 to Fig. 6 it is possible to draw conclusions on each of the equations in system Eq. (17), and to limit the domain of angular displacements where solutions can be found. The first equation of system Eq. (17) can be zero within the range $0 \leq \omega-\lambda_{\text {Sun }} \leq \pi$ and the total variation of eccentricity (second equation of system Eq. (17)) can be zero for $\pi \leq \omega-\lambda_{\text {Sun }} \leq 2 \pi$ (in both the equations, the limit values are for the case of SRP only). The Sun-synchronous condition $\Delta \omega_{\mathrm{SRP}, 2 \pi}=\Delta \lambda_{\mathrm{Sun}, 2 \pi}$ can be obtained for $\frac{\pi}{2} \leq \omega-\lambda_{\mathrm{Sun}} \leq \frac{3}{2} \pi$ (the limit values are for a circular orbit as can be seen in Fig. 6). Therefore, we can confirm that the system Eq. (17) cannot be satisfied if both solar radiation pressure and atmospheric drag are present. If the effect of drag is negligible, equilibrium orbits can be identified under the effect of solar radiation pressure for $\omega-\lambda_{\text {Sun }}=\pi$, as exploited by McInnes et al. [22] and Oyama et al. [23]. When solar radiation pressure and atmospheric drag both have a non-negligible effect on the spacecraft orbit, even if a complete equilibrium is not possible, it is useful to study partial equilibrium solutions:

$$
\begin{aligned}
& \left\{\begin{array}{l}
\Delta a_{\mathrm{SRP}, 2 \pi}+\Delta a_{\mathrm{Drag}, 2 \pi}=0 \\
\Delta \omega_{\mathrm{SRP}, 2 \pi}=\Delta \lambda_{\mathrm{Sun}, 2 \pi}
\end{array}\right. \\
& \left\{\begin{array}{l}
\Delta e_{\mathrm{SRP}, 2 \pi}+\Delta e_{\mathrm{Drag}, 2 \pi}=0 \\
\Delta \omega_{\mathrm{SRP}, 2 \pi}=\Delta \lambda_{\mathrm{Sun}, 2 \pi}
\end{array}\right.
\end{aligned}
$$

in which the Sun-synchronous condition is satisfied and only one variation, either semi-major axis or eccentricity is zero. These solutions are shown in Fig. 7 for SpaceChip 2 whose characteristics are reported in Table 1. Fig. 7a shows an example for which the total variation of semi-major axis (blue dotted line) due to SRP and drag is zero, and the Sun-synchronous condition is satisfied $\left(\Delta \omega_{\mathrm{SRP}, 2 \pi}\right.$ is represented by the continuous red line, $\Delta \lambda_{\mathrm{Sun}, 2 \pi}$ is represented by the continuous black bold line). Fig. 7b shows a solution of the system Eq. (19) for which the total variation of eccentricity (purple dashed line) due to SRP and drag is zero, and the Sun-synchronous condition is again satisfied. The exploitation of these partial equilibrium solutions of the systems Eq. (18) and Eq. (19) will be discussed later in Section V. 


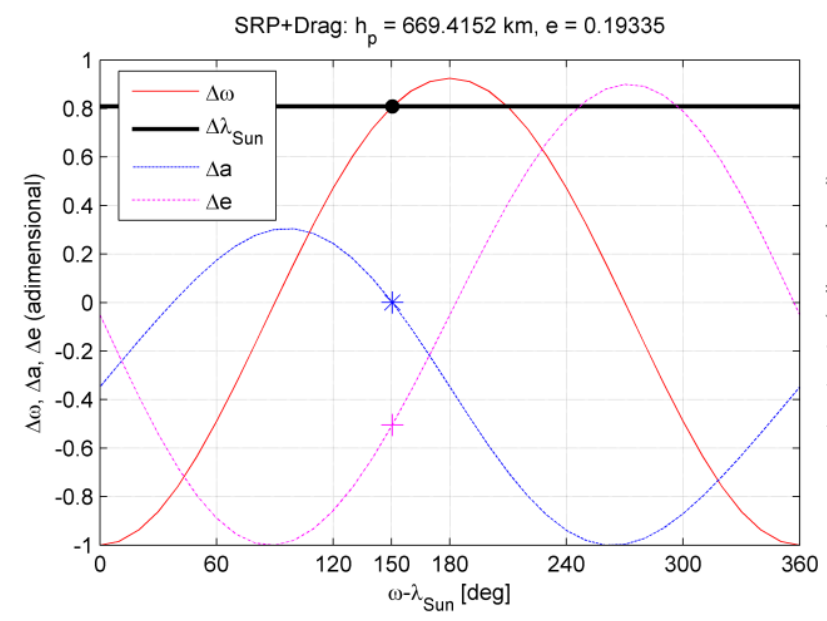

a)

Fig. 7 Variation of semi-major axis, eccentricity, anomaly of the pericentre over a single orbit revolution for SpaceChip 2 due to SRP and drag as a function of the initial condition in $\omega$ - $\lambda_{\text {Sun }}$. The variations of Keplerian elements were rescaled for ease of illustration. a) Condition for $\Delta a=0$ with the Sun-synchronous condition satisfied, and b) Condition for $\Delta e=0$ with the Sun-synchronous condition satisfied.

When only solar radiation pressure, without the Earth's shadow is considered the solution of system Eq. (17) can be determined analytically $[22,23]$. In the more complex case we consider, an analytical closed-form solution of systems Eqs. (17)-(19) was not possible; therefore, the problem is solved numerically. In this paper a global multistart approach is adopted. A local algorithm is started from several points randomly distributed over the entire domain of semi-major axis, eccentricity and arguments of angular displacement $\omega-\lambda_{\text {Sun }}$. For the results included in this paper we set $3.2 \times 10^{5}$ sampling points for the solution of each of the systems Eqs. (17)-(19). The numerical solution is made possible by the use of the semi-analytical approach explained in Section II $^{*}$. Alternatively, the use of the full numerical simulation for determining the change in orbital elements over a single orbit and the numerical solution of the eclipse geometry would make the computational time for the solution of systems Eqs. (17)-(19) impractical. Starting from each point on the mesh, a local minimization is performed numerically, through a subspace trust-region method, based on the interior-reflective Newton method [31, 32]. The objective function used for the minimization is:

$$
\min _{\left\{a, e, \omega-\lambda_{\text {Sun }}\right\}}\left[w_{a}\left|\Delta a_{\mathrm{Drag}, 2 \pi}+\Delta a_{\mathrm{SRP}, 2 \pi}\right|+w_{e}\left|\Delta e_{\mathrm{Drag}, 2 \pi}+\Delta e_{\mathrm{SRP}, 2 \pi}\right|+w_{\omega}\left|\Delta \omega_{\mathrm{SRP}, 2 \pi}-\Delta \lambda_{\mathrm{Sun}, 2 \pi}\right|\right]
$$

\footnotetext{
* The computational time for solving this problem was $13.5 \mathrm{~h}$ on one core of a processor Intel ${ }^{\circledR}$ Core ${ }^{\mathrm{TM}} 2$ Quad CPU Q9650 at $3.00 \mathrm{GHz}$.
} 
where $w_{a}, w_{e}, w_{\omega}$ are weight parameters introduced to treat this multi-objective minimization problem (the three equations of system Eqs. (17) must be solved) as a single-objective minimization. Depending on the value of the weight parameter, the multi-start algorithm can identify the solution set of system Eq. (17), Eq. (18), or Eq. (19). The ratio between $w_{a}$ and $w_{\omega}$, and $w_{e}$ and $w_{\omega}$ were chosen such that, for any solution found the Sun-synchronous apse-line condition is always satisfied. We restricted the eccentricity to be within the range $0.01 \leq e \leq 0.8$ which is of practical interest.

\section{Results for partial equilibrium orbits}

In the following section, the numerical results of the solution of systems Eq. (17), Eq. (18) and Eq. (19) will be shown, in the representation of the orbital element phase-space.

\section{A. Spacecraft and perturbation model}

We consider a silicon microchip with density $2.3 \mathrm{~g} / \mathrm{cm}^{3}$, and dimensions reported in Table 1 . Three different cases are analyzed, corresponding to increasing values of area-to-mass ratio. Table 1 reports the dimension of a microchip, $1 \mathrm{~cm}$ square, with different thickness values $l_{2}$, and for comparison, the radius $l$ of a sphere with an equivalent area-to-mass ratio. The spherical shape is usually adopted for studies of interplanetary dust dynamics. Since the SpaceChip density is assumed uniform, the characteristic length is represented by the chip's thickness:

$$
\begin{array}{rlrl}
A_{\text {chip }} & =l_{1}^{2} & & \frac{A_{\text {chip }}}{m_{\text {chip }}}=\frac{1}{l_{2} \cdot \rho_{\text {silicon }}} \\
m_{\text {chip }} & =l_{1}^{2} \cdot l_{2} \cdot \rho_{\text {silicon }} & & \\
A_{\text {sphere }} & =\pi l^{2} & & A_{\text {sphere }} \\
m_{\text {sphere }} & =\frac{4}{3} \pi l^{3} \cdot \rho_{\text {silicon }} & & m_{\text {sphere }} \\
\frac{4}{3} l \cdot \rho_{\text {silicon }}
\end{array}
$$

where $A$ is the cross-section area and $m$ the mass $^{\S}$. The size of SpaceChip 1 (see Table 1) was taken from the design by Atchison and Peck [7], hence it represents a near-term device. SpaceChip 2 and 3 represent scenarios with lower technology readiness levels, however they were selected to show the sensitivity of the conditions for long-lived orbits on the area-to-mass-ratio discussed later.

\footnotetext{
${ }^{\S}$ In the following we will omit the subscripts "chip" and "sphere" from $A$ and $m$ because the dynamical evolution of the 'smart dust' depends only on the area-to-mass ratio $\frac{A}{m}$, and not on its shape.
} 
In this paper we consider that the average effective cross-section $A_{\odot}$ exposed to the Sun is always equal to the cross-section area of the spacecraft $A$. This implies that the spacecraft has a spherical shape or its attitude is kept fixed with respect to the Sun-line. A passive Sun-pointing attitude control was proposed for millimeter-scale solar sails, based on faceted surfaces to stabilize the Sun-pointing plate [7]. Alternately, electro-chromic elements with variable reflectance can be layered at the sides of the chip to be exploited as a steering device, as demonstrated on the IKAROS mission [21]. Moreover, a reflectivity coefficient $c_{R}=1.8$ is assumed (note that for a black body $c_{R}=1$, for a flat mirror perpendicular to the light direction $c_{R}=2$ ).

For the model of atmospheric drag, a drag coefficient $c_{D}=2.1$ is chosen $\left(c_{D}=2.2\right.$ is usually used for a flat plate model, $c_{D}=2.0$ to 2.1 for spherical particles), and the cross-sectional area $A_{\text {Drag }}$ is considered constant and equal to the cross-section area of the spacecraft. The reference values for the computation of the air density in Eq. (13) where taken as [25]:

$$
\begin{aligned}
& h_{0}=600 \mathrm{~km} \\
& \rho_{0}=1.454 \times 10^{-13} \mathrm{~kg} / \mathrm{m}^{3} \\
& H=71.835 \mathrm{~km}
\end{aligned}
$$

since the region where solar radiation pressure and atmospheric drag are known to have comparable effect is approximately $400-800 \mathrm{~km}$. A more accurate value of the density would be obtained by selecting the reference values $h_{0}, \rho_{0}$ and $H$ closest to the range of orbit altitudes considered.

Table 1 also contains the characteristic acceleration due to solar radiation pressure computed through Eq. (2).

Note that a different choice of reflectivity or drag coefficient, or a different definition of cross-sectional area exposed to solar radiation or atmospheric drag, would change the numerical solution of the required initial conditions for equilibrium and long-lived orbit, but would not affect the overall concept proposed in this paper.

Table 1 SpaceChip characteristics.

\begin{tabular}{lllll}
\hline \hline & $\begin{array}{l}\text { Chip dimensions } \\
{[\mathrm{mm}]}\end{array}$ & $\begin{array}{l}\text { Sphere dimensions } \\
l[\mathrm{~mm}]\end{array}$ & $\mathrm{A} / \mathrm{m}\left[\mathrm{m}^{2} / \mathrm{kg}\right]$ & $a_{\mathrm{SRP}}\left[\mathrm{mm} / \mathrm{s}^{2}\right]$ \\
\hline SpaceChip 1 & $\begin{array}{l}l_{l}=10 \\
l_{2}=0.0250\end{array}$ & 0.0187 & 17.3913 & 0.1427 \\
\hline SpaceChip 2 & $\begin{array}{l}l_{l}=10 \\
l_{2}=0.0133\end{array}$ & 0.01 & 32.6087 & 0.2676 \\
\hline SpaceChip 3 & $\begin{array}{l}l_{1}=10 \\
l_{2}=0.00796\end{array}$ & 0.00597 & 54.6364 & 0.4484 \\
\hline \hline
\end{tabular}




\section{B. Analysis of long-lived orbits}

The solution of the systems Eqs. (17)-(19) can be represented in the phase space $\left\{\begin{array}{lll}e & \omega-\lambda_{\text {Sun }} & h_{p}\end{array}\right\}$ or

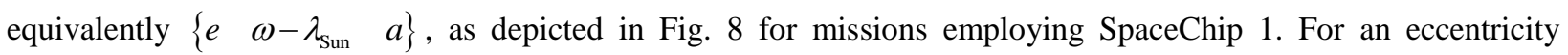
higher than approximately 0.115 and perigee altitude above $900 \mathrm{~km}$, a set of solutions for system Eq. (17) exists with the condition $\omega-\lambda_{\text {Sun }}=\pi$. With these initial conditions the orbit perigee is along the Sun-Earth direction, facing the Sun. This position is a stable condition for the variation of $\omega-\lambda_{\text {Sun }}$ and the change in eccentricity and semi-major axis over one orbit cancels, as the effect or SRP is symmetric in this configuration, and the drag is negligible (over one orbit). Considering the branch of the graph with $\omega-\lambda_{\text {Sun }}=\pi$, the greater the decrease of perigee altitude and eccentricity, the greater the effect of drag becomes up to a certain point at which it cannot be neglected. Beyond this point, as expected from the analysis in section III, no global equilibrium solutions (Eq. (17)) can be found. In this region (for perigee altitudes below $800 \mathrm{~km}$ approximately), atmospheric drag and SRP have comparable effects. However, a set of solutions still exist for system Eq. (18) (i.e., $\Delta a_{2 \pi}=0$ and the sunsynchronous condition satisfied) and Eq. (19) (i.e., $\Delta e_{2 \pi}=0$ and the sun-synchronous condition satisfied), represented respectively by the gray colored branch and the black colored branch of the graph. Note that each point in Fig. 8 corresponds to an initial condition for an Earth-centered orbit. Fig. 8 is therefore an example of a bifurcation. A solution for global equilibrium orbits (system Eq. (17)) can be found for eccentricities and perigee

altitudes higher than a certain value $\left\{\begin{array}{ll}e^{*} & h_{p}^{*}\end{array}\right\}$ (bifurcation point). Below the bifurcation point, the equilibria disappear and the only possible solution degrades to system Eq. (18) or Eq. (19) (only two out of the three equations of system Eq. (17) can be satisfied). 

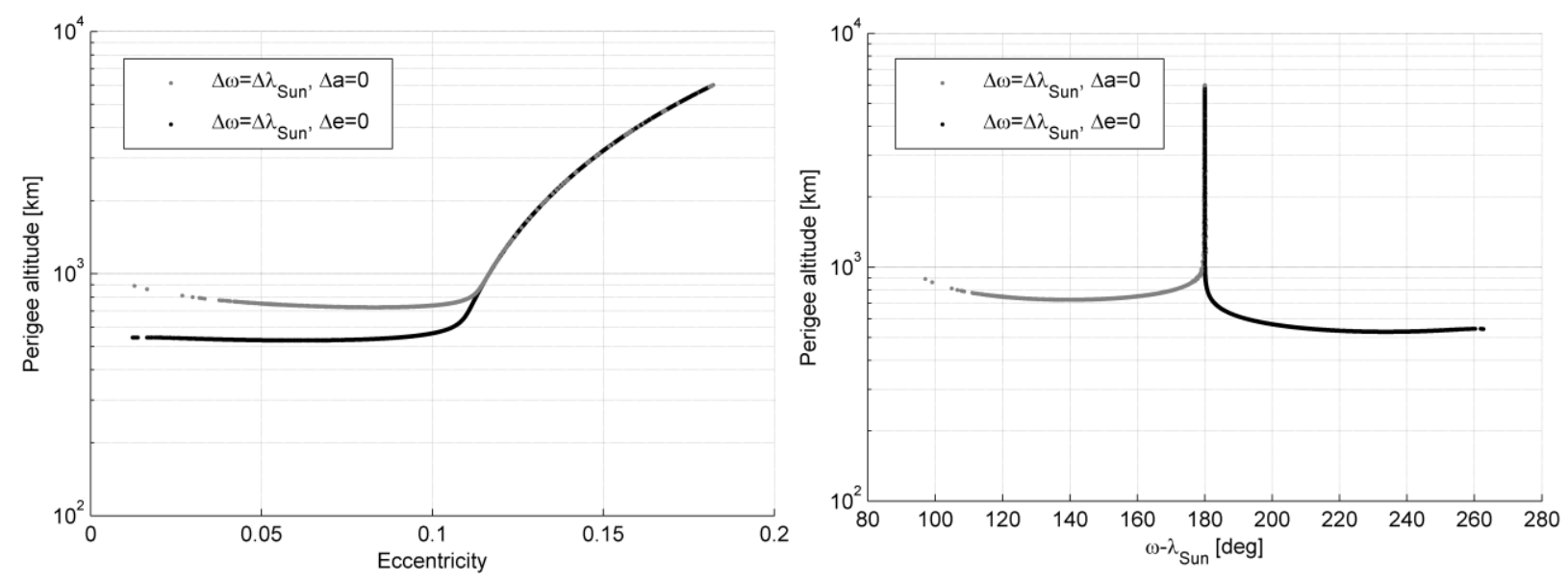

a)

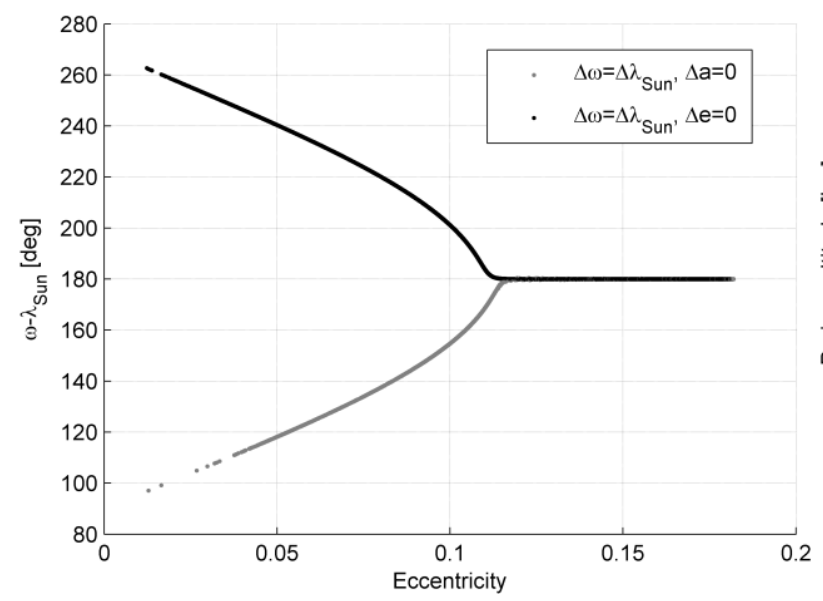

b)

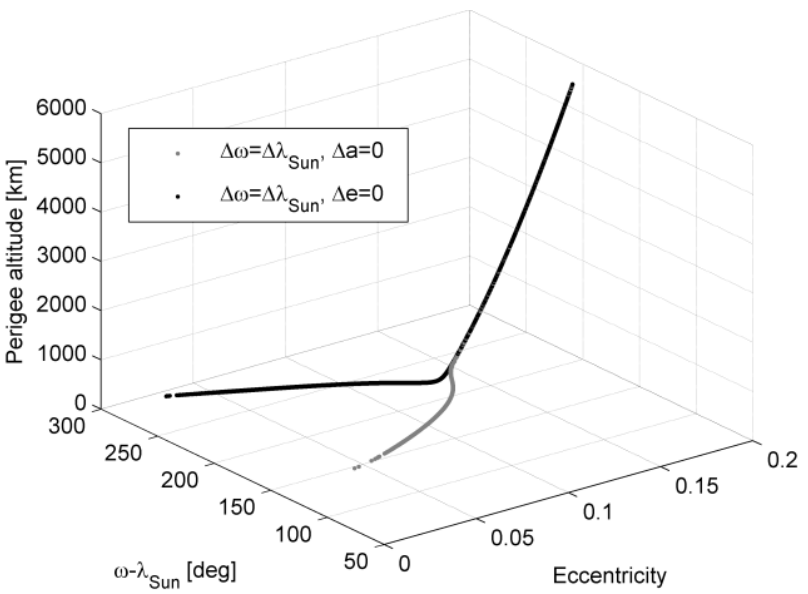

d)

c)

Fig. 8 Long-lived orbits conditions for SpaceChip 1 missions.

As expected, the lines in Fig. 8 represent the intersections between the surface solutions of system Eq. (17) as shown in Fig. 9. The surface $S_{1}$ is the solution of the Sun-synchronous condition $\Delta \omega_{\mathrm{SRP}, 2 \pi}=\Delta \lambda_{\mathrm{Sun}, 2 \pi}$, the surface $S_{3}$ on the domain $\pi \leq \omega-\lambda_{\text {Sun }} \leq 2 \pi$ contains the initial conditions for which the variation of the eccentricity due to SRP and drag balances (i.e., $\Delta e_{\mathrm{SRP}, 2 \pi}+\Delta e_{\mathrm{Drag}, 2 \pi}=0$ ) and the surface $S_{2}$ on the domain $0 \leq \omega-\lambda_{\text {Sun }} \leq \pi$ represents the solution of $\Delta a_{\mathrm{SRP}, 2 \pi}+\Delta a_{\mathrm{Drag}, 2 \pi}=0$. In correspondence with the condition $\omega-\lambda_{\mathrm{Sun}}=\pi$, above a certain value of the perigee altitude, the surfaces $\Delta e_{\mathrm{SRP}, 2 \pi}+\Delta e_{\mathrm{Drag}, 2 \pi}=0$ and $\Delta a_{\mathrm{SRP}, 2 \pi}+\Delta a_{\mathrm{Drag}, 2 \pi}=0$ intersect as drag becomes negligible and the orbit precesses due to SRP alone (line C).

Both the perturbing acceleration due to solar radiation and atmospheric drag are directly proportional to the areato-mass ratio of the spacecraft (see Eq. (2) and Eq. (12)), hence we can expect that the surfaces 
$\Delta a_{\mathrm{SRP}, 2 \pi}+\Delta a_{\mathrm{Drag}, 2 \pi}=0$ and $\Delta e_{\mathrm{SRP}, 2 \pi}+\Delta e_{\mathrm{Drag}, 2 \pi}=0$ remain unchanged for any area-to-mass ratio. Instead, only the surface $\Delta \omega_{\mathrm{SRP}}-\Delta \lambda_{\mathrm{Sun}, 2 \pi}=0$ will change.
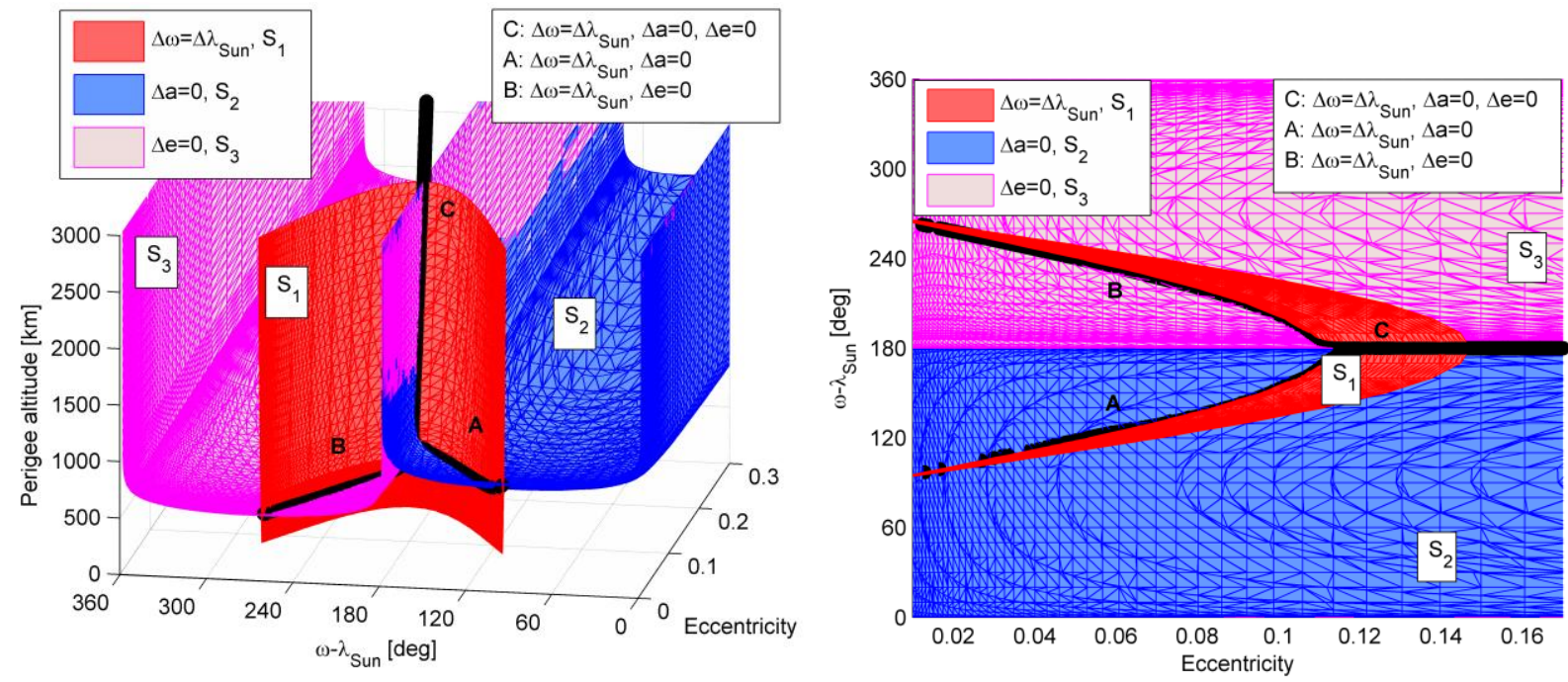

a)

b)

Fig. 9 Surfaces representing each of the equations of system Eq. (17) for SpaceChip 1 missions. a) 3D view, and b) 2D view in eccentricity $-\omega-\lambda_{\text {Sun }}$.

In Fig. 10 the solutions for different SpaceChip dimensions are shown to demonstrate the sensitivity of the solution to the spacecraft area-to-mass ratio. In particular, when the parameter $\frac{A}{m}$ increases, the amplitude of $\Delta \omega_{\mathrm{SRP}, 2 \pi}$, represented in Fig. 6, increases, but $\Delta \lambda_{\text {Sun }}$ is unchanged since it does not depend on the spacecraft characteristics. Therefore, the intersection surface, shown in Fig. 9, is displaced for increasing values of eccentricity, as can be seen in Fig. 10. On the other hand, the more the area-to-mass ratio decreases, the smaller will be the effect of drag and SRP with respect to the gravitational attraction of the Earth. The equilibrium solution corresponding to $\omega-\lambda_{\text {Sun }}=\pi$ can be found at higher values of semi-major axis, however in the case of small conventional spacecraft (e.g., CubeSats) the range of values of semi-major axis at which the Sun-synchronous condition is satisfied is so high to be impractical and out of the range of validity of the dynamical model considered.

For a higher (or lower) value of the reflectivity coefficient the surface $\Delta \omega_{\mathrm{SRP}}-\Delta \lambda_{\mathrm{Sun}, 2 \pi}=0$ would be displaced in the phase space at higher (or lower) eccentricities, and if the ballistic coefficient remain constant, the surfaces 
$\Delta a_{\mathrm{SRP}, 2 \pi}+\Delta a_{\text {Drag, } 2 \pi}=0$ and $\Delta e_{\mathrm{SRP}, 2 \pi}+\Delta e_{\text {Drag, } 2 \pi}=0$ would be displaced at lower (or higher) perigee altitudes [33].

Similarly, the increase (or decrease) of ballistic coefficient for the same reflectivity coefficient would cause the displacement of the equilibrium surfaces of semi-major axis and eccentricity because the equilibrium between SRP and drag would move at higher (or lower) orbit altitudes.
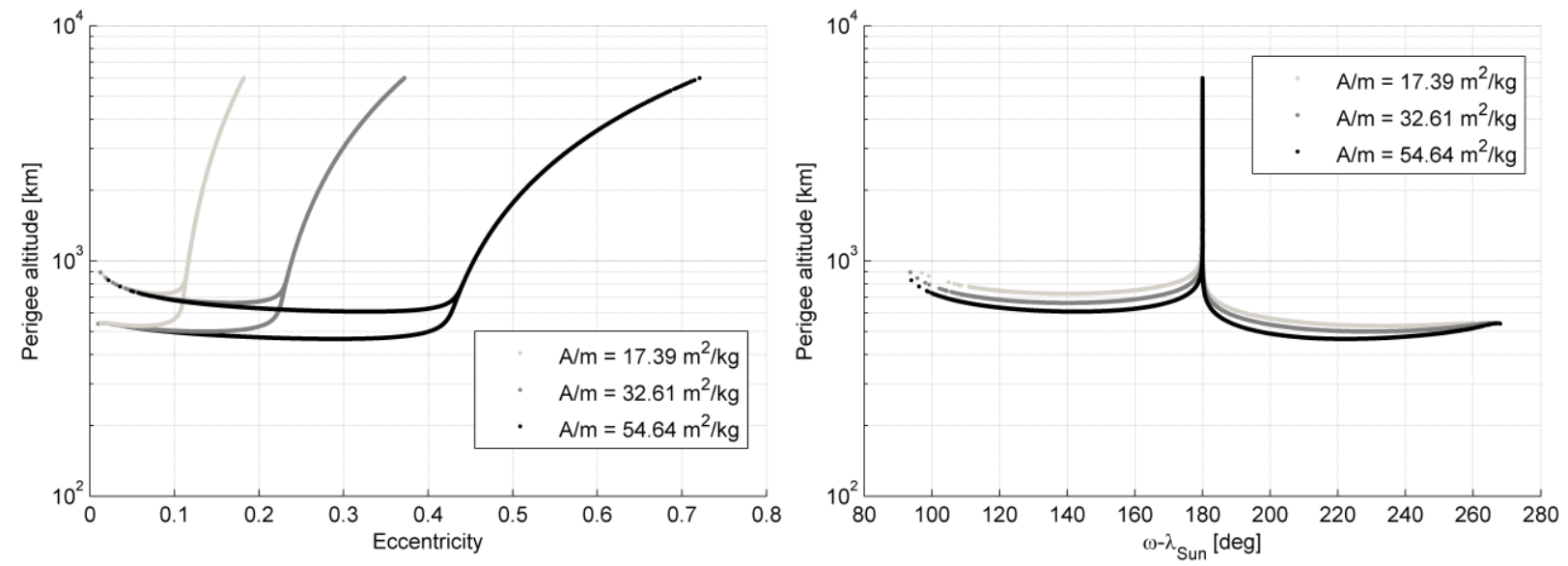

a)

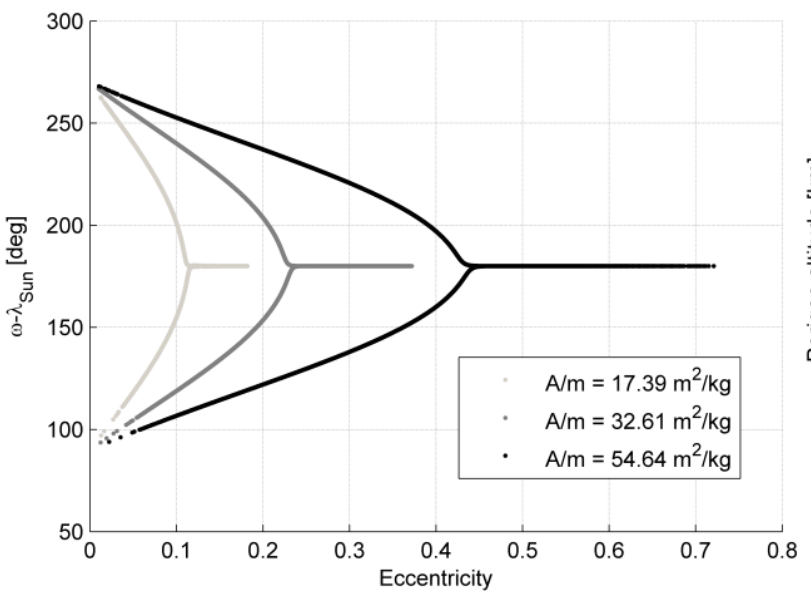

b)

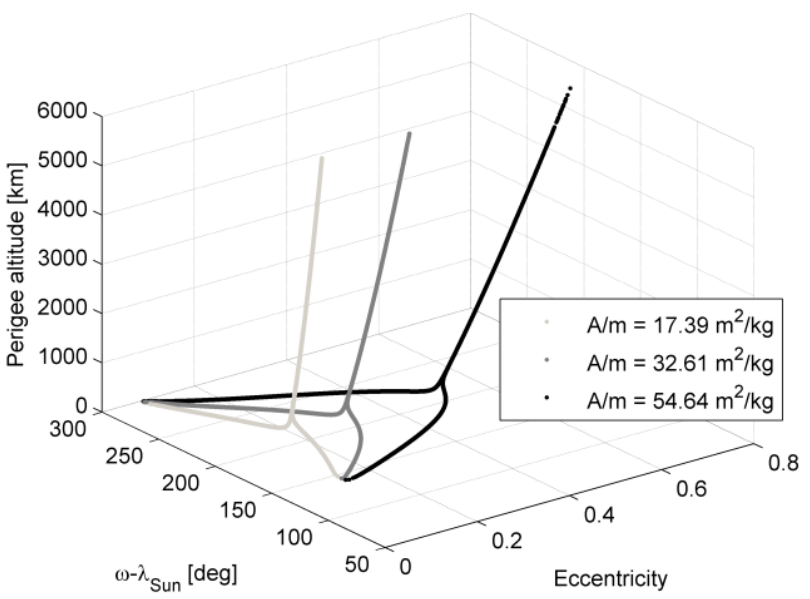

c)

d) 


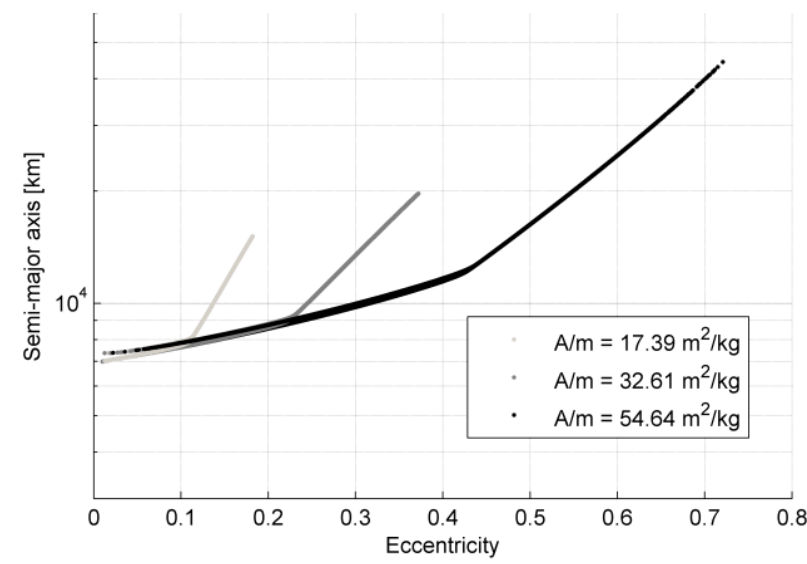

e)

Fig. 10 Dependence of conditions for long-lived SpaceChip orbits on orbital parameters and area-tomass ratio.

In the following sections we will refer to Fig. 8 and Fig. 10 as long-lived orbits surfaces and we will indicate the Keplerian tuple of initial conditions (i.e., each point on the long-lived orbits surface) with the symbol $k_{L L}$. Note that in the following $k e p_{L L}$ will be also called vector of initial conditions, even if we are aware that a set of Keplerian elements is not a vector but a tuple because it does not satisfy the transformation rules of a vector (and has not the physical meaning of a vector).

\section{Long-term orbit evolution}

In this section we analyze the long-term evolution of orbits whose initial conditions are represented by the set of points in Fig. 8, and Fig. 10. The long-term evolution is predicted by integrating Eqs. (16) and using a stopping condition for the integration:

$$
C_{\text {forw }}:\left\{h_{p} \leq 250 \mathrm{~km} \vee e \leq 0.01 \vee e \geq 0.8\right\}
$$

that is when the orbit perigee drops under a set limit of $250 \mathrm{~km}$ or the eccentricity exceeds the lower or upper bounds of 0.01 and 0.8 , which is our domain of interest. The first condition of Eqs. (20) is set because the orbit rapidly decays below a certain perigee altitude and the mission is over. Later in this section, it will be useful to propagate the initial conditions backwards in time, to find the foregoing behavior of the spacecraft. In this case the stopping condition for the integration is:

Journal of Journal of Guidance, Control and Dynamics 


$$
C_{\text {back }}:\left\{h_{p} \leq 250 \mathrm{~km} \vee h_{p} \geq h_{p, \text { up }} \vee e \leq 0.01 \vee e \geq 0.8\right\}
$$

where an upper limit on the perigee altitude $h_{p \text {, up }}$ is introduced. The numerical integration of Eqs. (16) is performed through an adaptive step-size Runge-Kutta-Fehlberg integration scheme integrator with a six stage pair of approximation of the fourth and fifth order [34], with absolute and relative tolerance of $2.5 \times 10^{-14}$.

For a better understanding of the long-term behavior, it is useful to distinguish two different parts of the longlived orbit condition graph. We indicate with $e^{*}$ the value of the eccentricity at which the bifurcation takes place. Note that the exact value of $e^{*}$ depends on the tolerances set for the solution of system Eq. (17), which determine when the solution for a stable orbit (i.e., three equations of system Eq. (17) satisfied) does not exist anymore. In this case we can still find partial equilibrium solutions represented by systems Eq. (18) and Eq. (19). According to this definition we define the sections of long-lived orbits surface as:

- $e<e^{*}$ where we can identify the two branches of the solutions sets of system Eq. (18) and Eq. (19);

- $e \geq e^{*}$ where the two branches progressively merge in the solution of system Eq. (17).

\section{A. Region $e<e^{*}$}

We now focus on the first region of the solution space, in particular on the branch representing the solutions of system Eq. (18) (i.e., $\Delta a_{2 \pi}=0$ and the Sun-synchronous condition satisfied). The dynamics of the SpaceChip have been propagated, starting from some vector of initial conditions belonging to this set. For each initial point, the orbit has been propagated backward in time, considering the dynamics in the presence of SRP and drag (Eqs. (16)), until one of the conditions in Eq. (21) is met. Then, the final state of the backward integration is used as the initial condition for the forward integration, until one of the conditions in Eq. (20) is met. Having the highest value of areato-mass ratio, the effect of the perturbations is most pronounced for SpaceChip 3, hence we select this scenario for the analysis in this section. In Fig. 11 the long-term evolution subject to SRP and drag is shown with the black line, starting from the initial state indicated with the black symbol '+', until the stopping criterion Eq. (20) is met. The behavior is similar for any vector of initial conditions in the set. The orbit perigee drifts following the apparent Sunline rotation, starting behind the Sun and moving ahead of it while the perigee altitude rises when $0<\omega-\lambda_{\text {Sun }}<\pi$ and decreases when $\pi<\omega-\lambda_{\text {Sun }}<2 \pi$. Each line bends right in correspondence to the branch with $\Delta e_{2 \pi}=0$ and $\Delta \omega_{2 \pi}=\Delta \lambda_{\text {Sun, } 2 \pi}$, and then the spacecraft orbit evolves towards decay. In this case all the end-points represented by 
the black dot symbol are in correspondence with the stopping condition $h_{p}=250 \mathrm{~km}$ of Eq. (20). In Fig. 11 the orbit evolution under the effect of drag only is superimposed (gray line), starting from the same initial conditions of the SRP and drag case. The orbit shrinks while the radius of the perigee tends to remain constant (see Fig. 11c). The difference between the SRP and drag and drag-only case is highlighted in Fig. 11c-d. Notably, the effect of SRP causes a significant increase in the orbit lifetime with respect to the drag-only case, as shown in Fig. 12, as a function of the eccentricity of the initial condition vector on the long-lived orbits surface ('o' symbol in Fig. 11). The orientation of the orbit apse-line relative to the Earth's shadow leads to a gain in orbit energy to balance the dissipation due to air drag, as evidenced by the looping trajectory in the orbital element space.

The long-term forward evolution of the orbits with initial conditions which lie on the branch representing the solutions of system Eq. (19) (i.e., $\Delta e_{2 \pi}=0$ and Sun-synchronous condition satisfied), instead, leads to a fast orbit decay, and the presence of SRP contributes to a decrease of the orbit lifetime with respect to the drag-only case (see Fig. 13 and Fig. 14). This is shown in Fig. 13, where the black line represents the SRP and drag scenario, the gray line the drag-only scenario and the initial condition belongs to the gray branch $\left(\Delta e_{2 \pi}=0\right.$ and $\Delta \omega_{2 \pi}=\Delta \lambda_{\text {Sun, } 2 \pi}$ ). Note that in the SRP and drag case, the orbit decay phase does not evolve at a constant perigee radius, as in the case of drag only. Moreover, the gray lines in Fig. 13b (drag-only case) which jump from $\omega-\lambda_{\text {Sun }}=0$ to $\omega-\lambda_{\text {Sun }}=2 \pi$ correspond to rotational motion, where $\omega-\lambda_{\text {Sun }}$ continues to decrease.
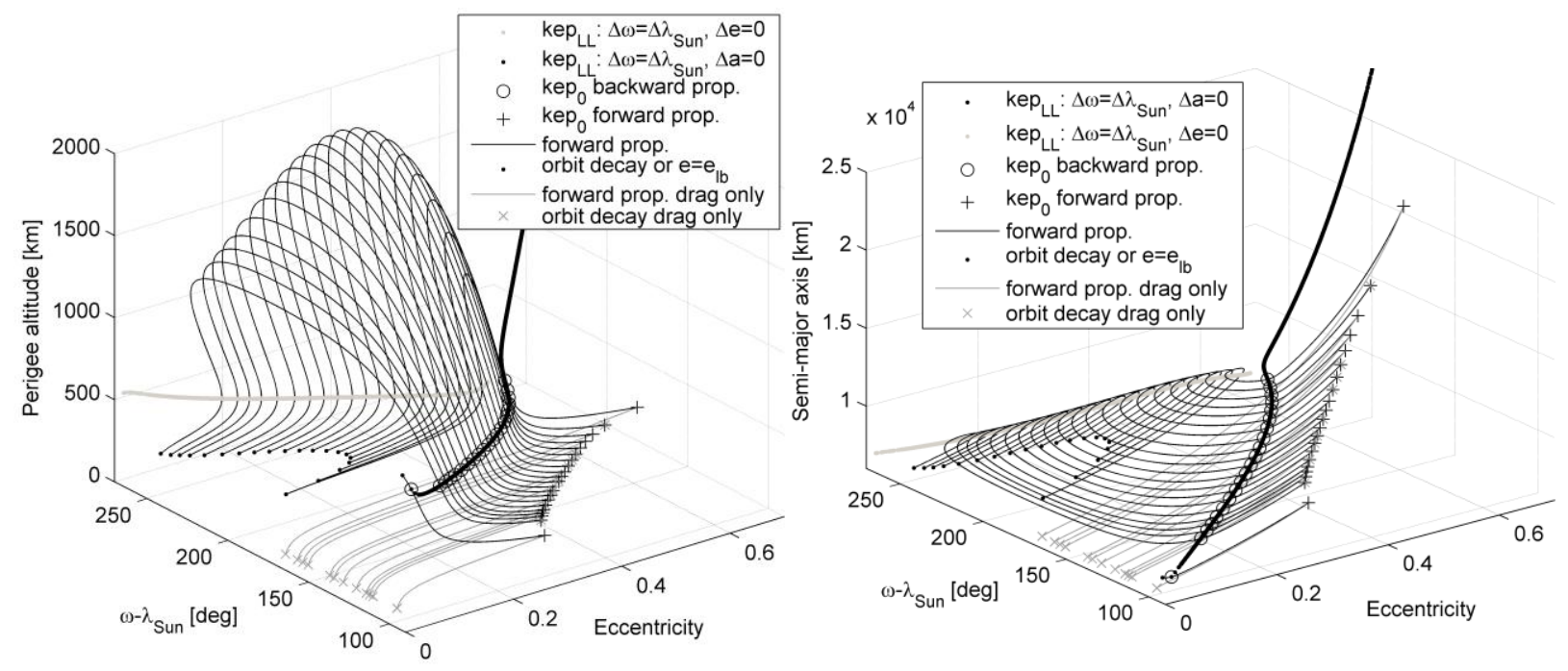

a)

b)

Journal of Journal of Guidance, Control and Dynamics 

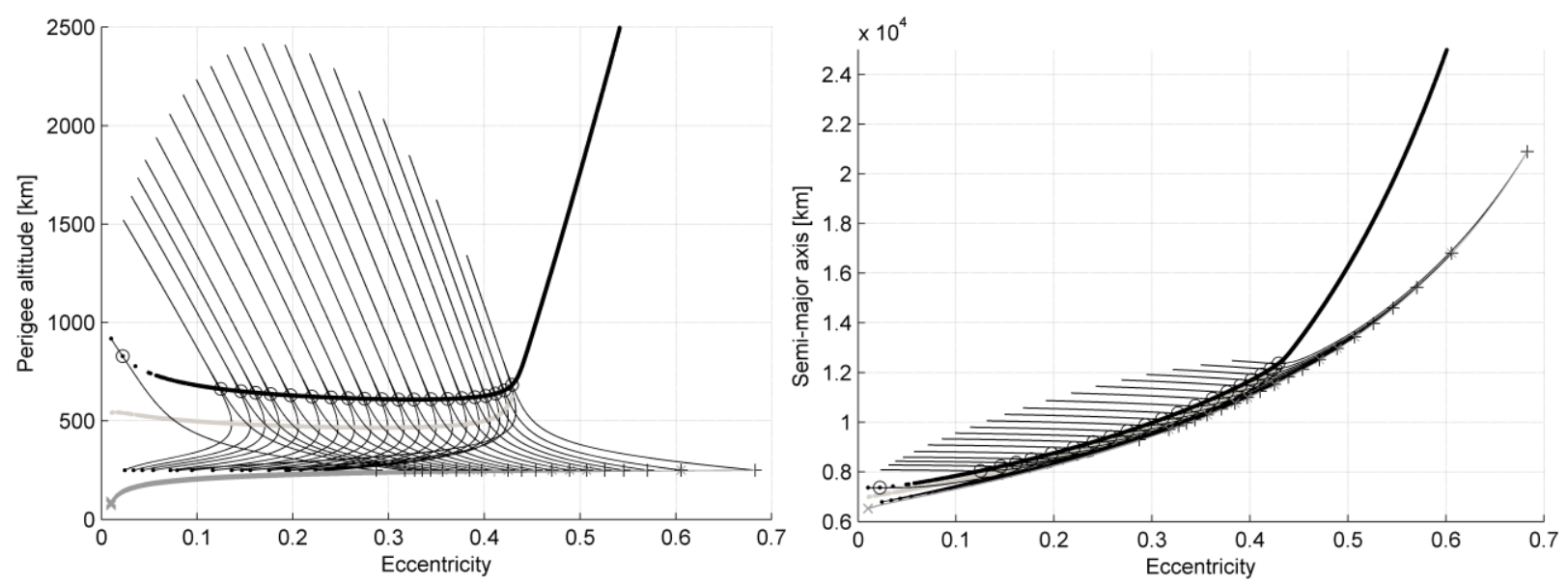

c)

d)

Fig. 11 Long-term orbit evolution for SpaceChip 3 from initial conditions with $\Delta a=0$ and $\Delta \omega=\Delta \lambda_{\text {Sun }}$. The black lines represent the SRP and drag case, the gray lines the drag-only case. a) 3D view in the phase-space with perigee altitude on the $z$-axis, b) $3 D$ view in the phase-space with semi-major axis on the z-axis, c) 2D view in perigee altitude-eccentricity, d) 2D view in semi-major axis-eccentricity.

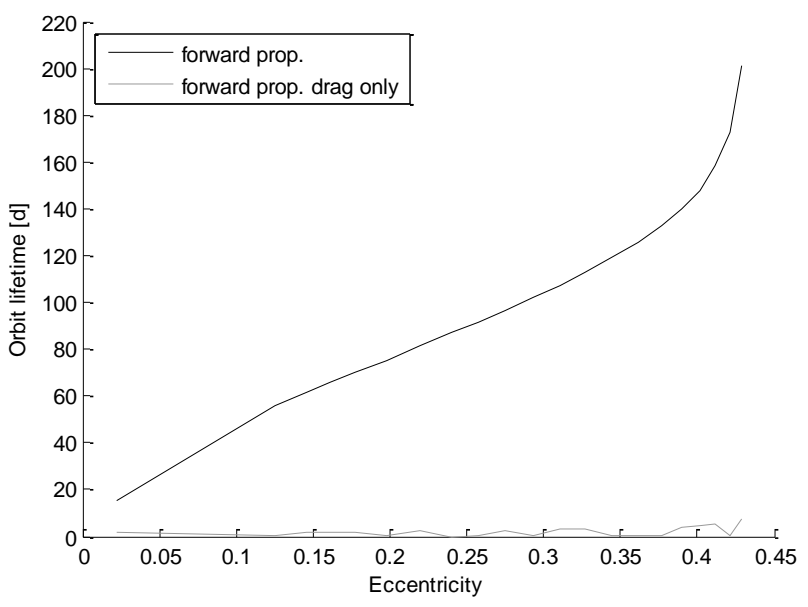

Fig. 12 SpaceChip 3 orbit lifetime for evolution from initial conditions with $\Delta a=0$ and $\Delta \omega=\Delta \lambda_{\text {Sun. }}$ The black line represents the SRP and drag case, the gray line the drag-only case. 

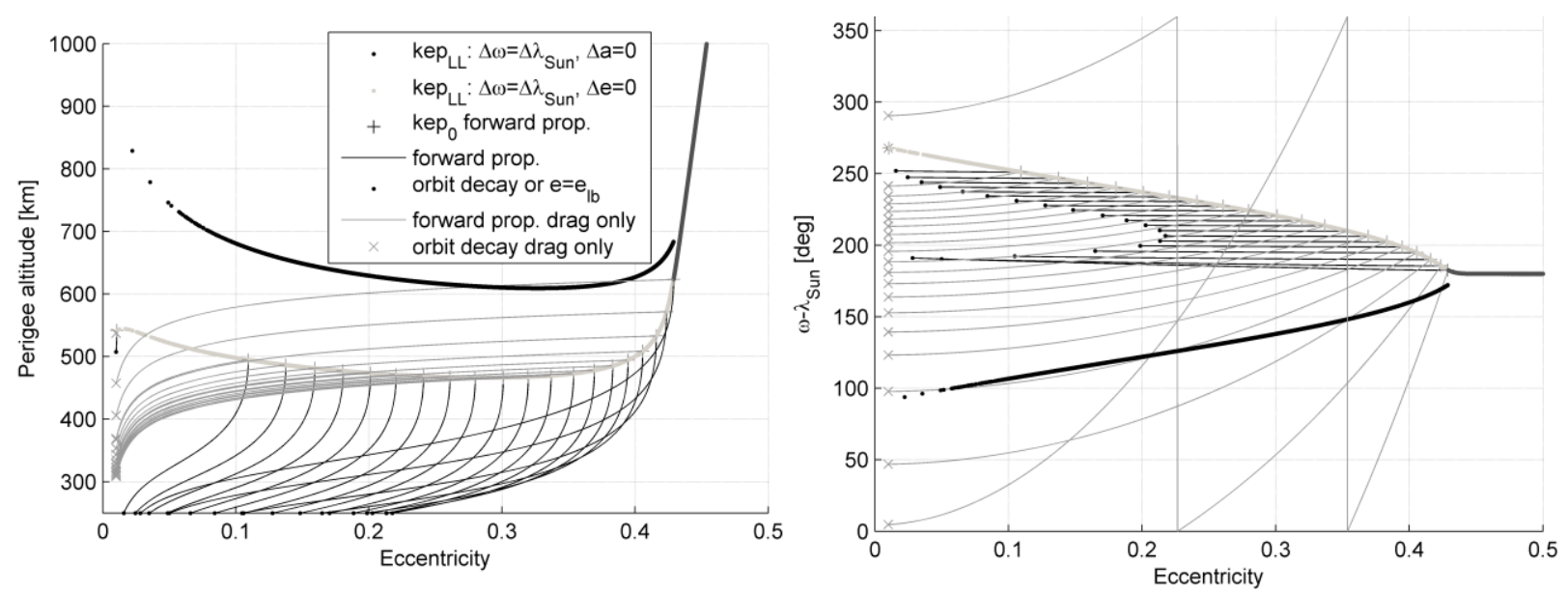

a)

b)

Fig. 13 Long-term orbit evolution for SpaceChip 3 from initial conditions with $\Delta e=0$ and $\Delta \omega=\Delta \lambda_{\text {Sun }}$. The black lines represent the SRP and drag case, the gray lines the drag-only case.

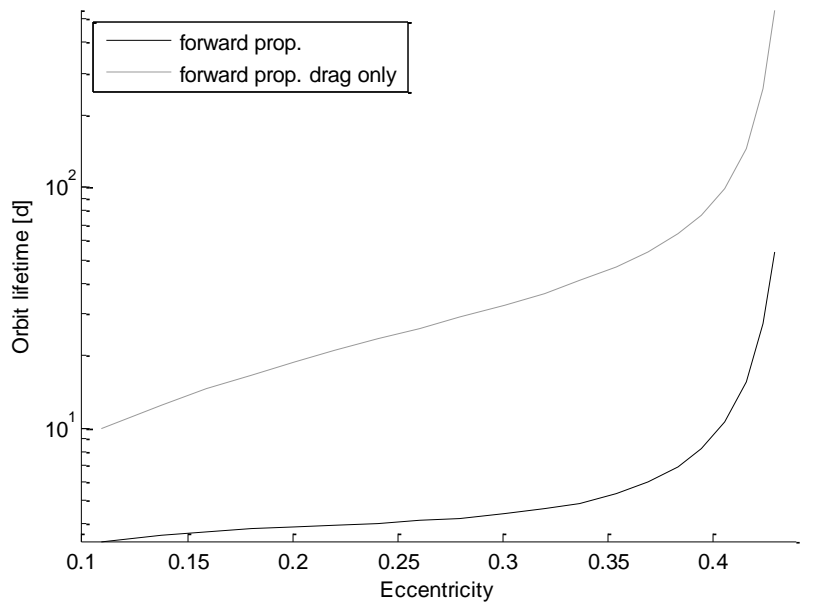

Fig. 14 SpaceChip 3 orbit lifetime for evolution from initial conditions with $\Delta e=0$ and $\Delta \omega=\Delta \lambda_{\text {Sun }}$ The vertical axis is in logarithmic scale. The black line represents the SRP and drag case, the gray line the dragonly case.

\section{B. Region $e \geq e^{*}$}

When we consider an initial condition vector which straddles the bifurcation region, the long-term evolution of the orbit presents interesting behavior. As before, it is convenient to propagate the dynamics backwards in time, starting from a vector of initial conditions selected from the set of solutions of the system Eq. (17) or of the system Eq. (18) with a value of eccentricity close to the bifurcation region. The backward integration is continued until the 
stopping condition Eq. (21) is met. Fig. 15 shows an example of this orbit evolution behavior. The selected condition on the long-lived orbit graph is:

$$
k e p_{L L}: e=0.44085, h_{p}=799.0144 \mathrm{~km}, a=12835.8 \mathrm{~km}, \omega-\lambda_{\text {Sun }}=178.5229 \mathrm{deg}
$$

and $h_{p \text {, up }}$ was fixed at $3000 \mathrm{~km}$. Note that the choice of $h_{p \text {,up }}$ is arbitrary, as it is set only to fix a limit on the backward integration. The final state of the backward integration (black '+' symbol in Fig. 15) is used as the initial condition for the forward integration, until one of the conditions in Eq. (20) is met. As can be seen from Fig. 15a and the two projections in Fig. 15b and c, the long-term evolution in the phase-space diagram is characterized by a librational and progressively decaying motion around the equilibrium line which represents the solution of system Eq. (17); i.e., the spacecraft performs quasi-closed loops in the eccentricity- $\left[\omega-\lambda_{\text {Sun }}\right]$ plane around the equilibrium point at which $\left\{\Delta a_{2 \pi}=0, \Delta e_{2 \pi}=0, \Delta \omega_{2 \pi}=\Delta \lambda_{\text {Sun, } 2 \pi}\right\}$. With respect to the SRP-only case (see Ref. [23]) the loops do not close completely as they become smaller, while their centre point moves along the equilibrium line towards increasing values of eccentricity, i.e., the spacecraft describes a spiral in the orbital element phase-space. The orbit perigee oscillates around the Sun-line while the orbit stretches and contracts due to the oscillation both in eccentricity and semi-major axis. Fig. 16 depicts the evolution under SRP and drag in terms of orbit shape. Over one single loop in Fig. 15, the orbit librates as represented in Fig. 16a (orbit 1, orbit 2, orbit 3, orbit 4). Due to the effect of drag, a constant decaying motion is superimposed on the librational motion (see Fig. $16 \mathrm{~b}$ orbit 5, orbit 6, orbit 7 , orbit 8 ). This is clearly visible in Fig. 15b and Fig. 15d; the librational loops (due to the effects of SRP) become progressively smaller (i.e., the deviation from the centre value of eccentricity, $\omega-\lambda_{\text {sun }}$, and perigee altitude decreases) due to the effect of atmospheric drag. In particular from Fig. 15e, it is possible to infer that the effect of drag is almost negligible over the major part of the librational loop and becomes predominant in the arc of the loop when the perigee reaches its local minimum. In correspondence to the local minima of the perigee altitude, which oscillates due to SRP, the spacecraft experiences a rapid drop in orbit energy (see the step-fall in the semi-major axis in Fig. 15e), therefore the following librational loop will be centered on a point with a lower semi-major axis (i.e., lower value of the averaged orbit energy over the overall librational loop). In Fig. 15e the forward propagation due to SRP and drag (black line) is compared with the orbit evolution subjected to SRP only (bold black line). In the latter case the motion is exclusively librational (i.e., the spacecraft perpetually travels over the initial loop). Note that 
the inclination of the librational loop under SRP only, visible in the eccentricity - semi-major axis projection, is due to the asymmetry in geometry due to eclipses. In fact, in the presence of Earth's shadow the secular variation of semi-major axis is different than zero; therefore, the orbit energy is not conserved but the average energy, over one librational loop, is conserved.

The higher the equilibrium value of the semi-major axis (i.e., centre point of the librational loop), the lower is the excursion in semi-major axis over one loop. For a value of semi-major axis sufficiently high, the effect of eclipses can be neglected, and the SRP model (with eclipses) reduces to the model used by Oyama et. al. (without eclipses) for which $\frac{d \bar{a}}{d t}{ }_{\text {SRP }}$ can be considered to be zero [23].

In Fig. 15 the orbit evolution under the effect of drag only is shown (gray line). The initial condition for the forward propagation was set equal to the point of the forward propagation with SRP and drag (black line) where the first local minimum of perigee altitude is reached (gray ' + ' symbol).

When the effect of SRP cannot be exploited $\left(c_{R}=0\right)$, the orbit evolution follows a rotational motion where $\omega-\lambda_{\text {Sun }}$ continuously decreases (see Fig. 15a and Fig. 15c). The evolution of eccentricity and perigee altitude are also different; the orbit becomes increasingly circular while the perigee altitudes stays almost constant (see Fig. 15b). Once the orbit eccentricity becomes zero, the orbit radius starts to shrink quickly until the final decay in the lower atmosphere (gray ' $x$ ' symbol). In the SRP and drag scenario the final (and very fast) leg of the orbit decay lies within the $\omega-\lambda_{\text {Sun }}$ domain bounded by the two branches of the solutions of systems Eq. (18) and Eq. (19). Therefore, the bifurcation region can be seen as a sink for all the librational motion trajectories that design at higher values of semi-major axis.

The oscillation in perigee and apogee altitude through the orbit evolution is shown in Fig. 15f (black line) and is compared to the drag-only scenario (progressive constant decrease of the apogee, while the perigee altitude tends to remain constant), represented by the continuous gray line, and the SRP-only scenario (the apogee and perigee continuously oscillate between their minimum and maximum value), represented by the bold black line.

Finally, Fig. 17 compares the time evolution of the Keplerian elements, under the effect of SRP and drag (black line), drag only (gray line), and SRP only (light gray line). The perigee oscillation due to SRP is damped by the presence of drag (see Fig. 17a). The atmospheric drag, mainly experienced in the region around $700 \mathrm{~km}$ (for SpaceChip 3), causes the decrease of the maximum value of the perigee that is reached over each librational loop (in 
analogy with the orbit apogee of the drag-only case, see Ref. [25] Fig. 9-11 p. 674). The minimum perigee (in analogy with the orbit perigee of the drag-only case), instead, tends to remain constant until the last part of the decay phase. The semi-major axis behavior in time is characterized by a periodic motion (with the period of one librational loop that is different from the period of one single orbit) due to SRP, plus a quasi-constant energy decrease due to drag (see Fig. 17b). Note that the orbit lifetime with SRP and drag is around 28 years, compared to the 11.3 years of the drag-only scenario. Therefore, long-lived orbits can be designed where asymmetric solar radiation pressure can balance energy dissipation due to air drag. In addition, the effect of atmospheric drag can be exploited to ensure the end-of-life decay of SpaceChips, thus preventing long-lived orbit debris. As already pointed out for Fig. 15d, the orbit perigee oscillates around the Sun direction $\left(\omega-\lambda_{\text {Sun }}=\pi\right)$ and the oscillation are damped by atmospheric drag (see Fig. 17c). In the drag-only scenario, $\omega-\lambda_{\text {sun }}$ is continuously decreasing as shown in Fig. 15a and Fig. 15c. As can be seen in Fig. 17d and Fig. 15b, the eccentricity oscillates around the value that satisfies system Eq. (17) for a given semi-major axis and $\omega-\lambda_{\text {sun }}=\pi$.
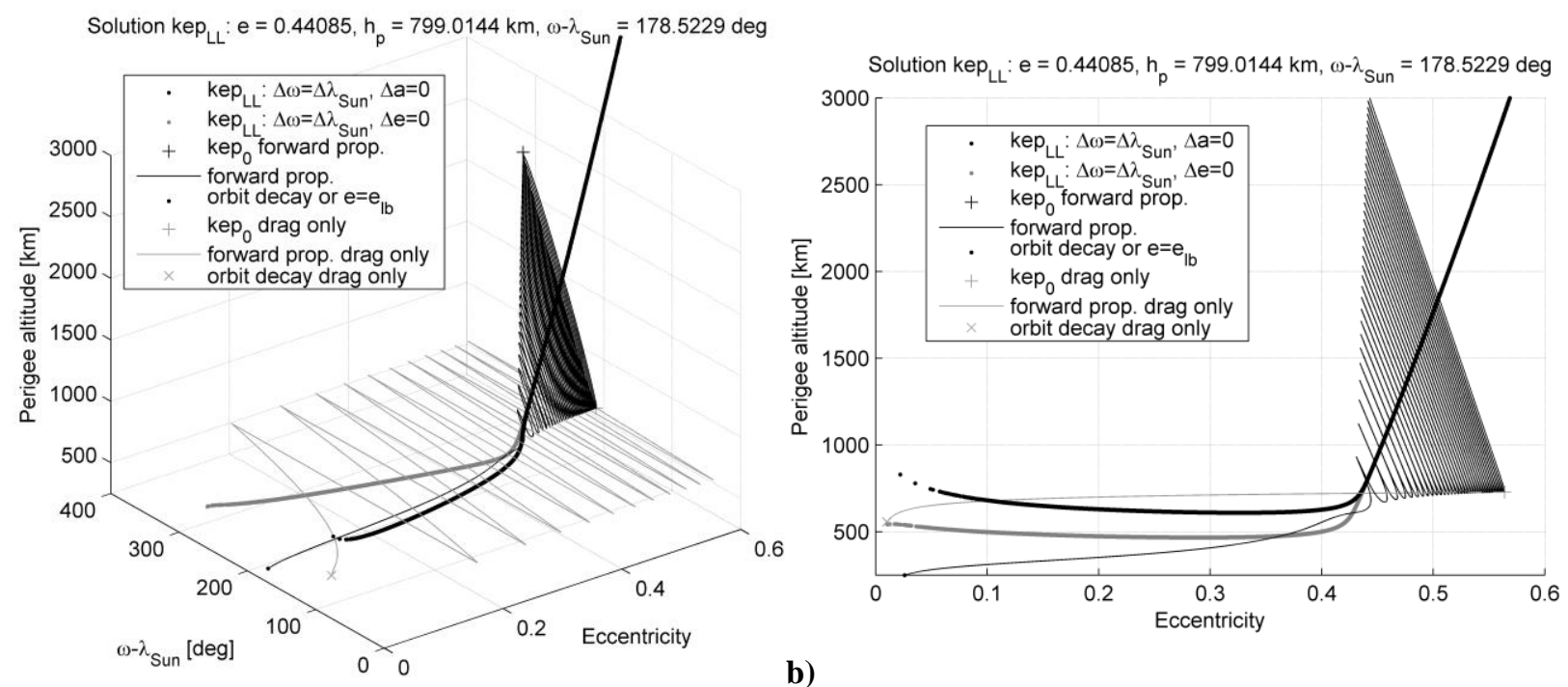

a)

b) 

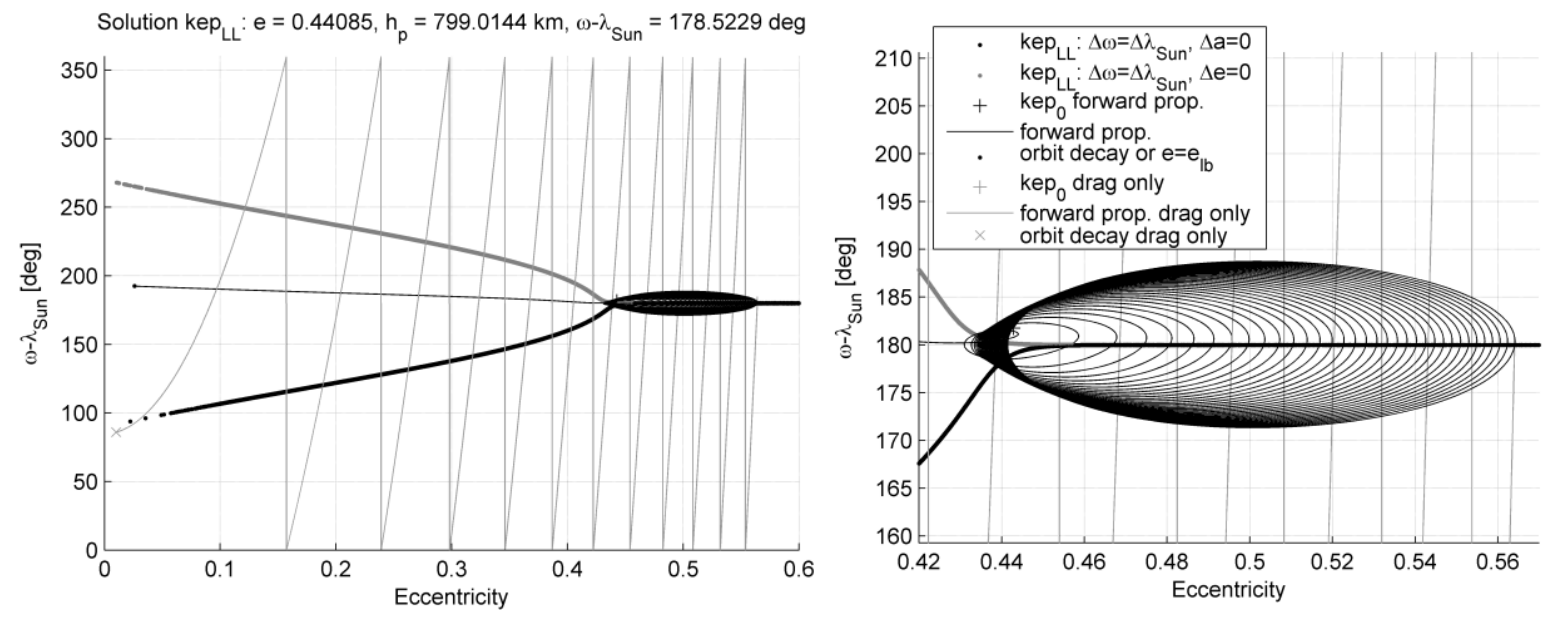

c)

d)
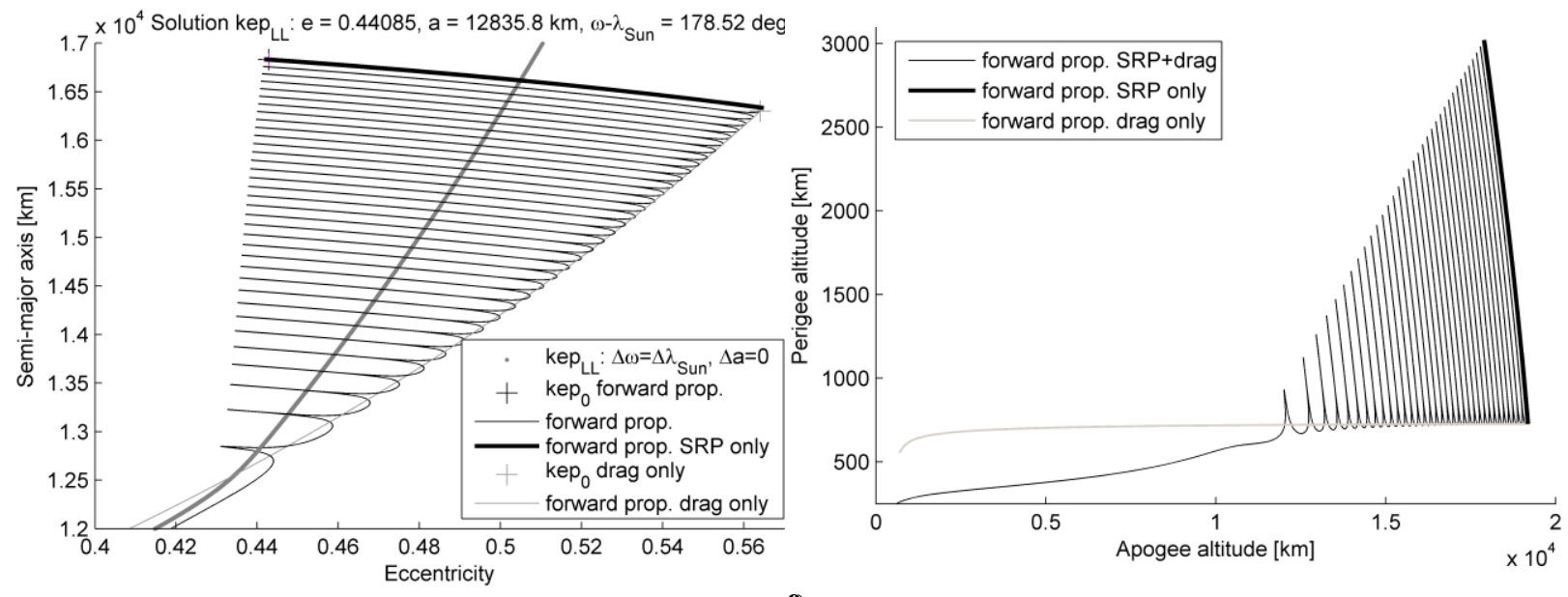

e)

f)

Fig. 15 Long-term orbit evolution for SpaceChip 3 from a vector of initial conditions for librational motion under the influence of SRP and drag (black lines), drag only (gray lines), and SRP only (bold black lines). a) 3D view in the phase-space, b) 2D view in eccentricity - perigee altitude, c) 2D view in eccentricity $-\omega-\lambda_{\text {Sun }}$, d) zoom on the librational motion, e) 2D view in eccentricity - semi-major axis, and f) oscillation of the apogee and the perigee altitude. 

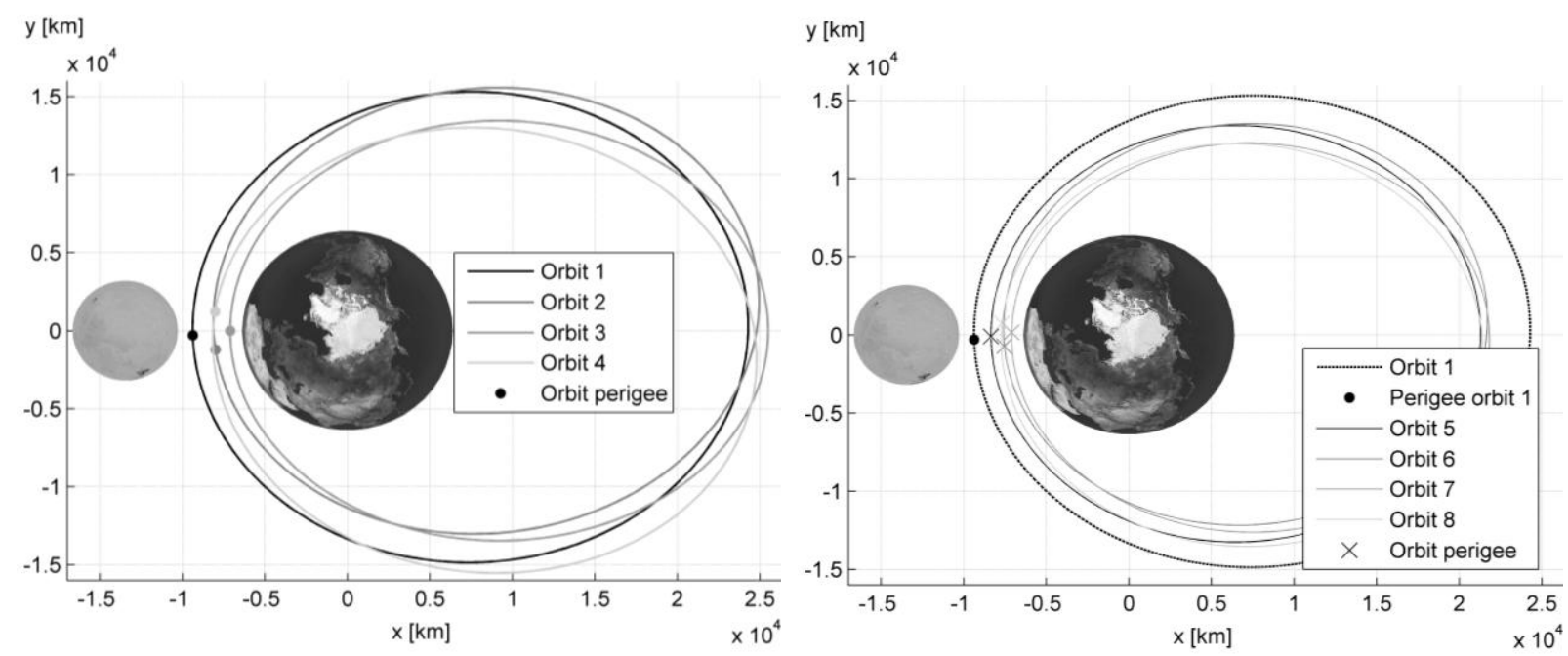

a)

b)

Fig. 16 Librational and progressively decaying motion due to SRP and drag. a) The orbit perigee oscillates around the Sun-line and the orbit shape changes due to the oscillation in eccentricity and semimajor axis. b) Due to the effect of drag the orbit perigee is subject to a secular decrease.
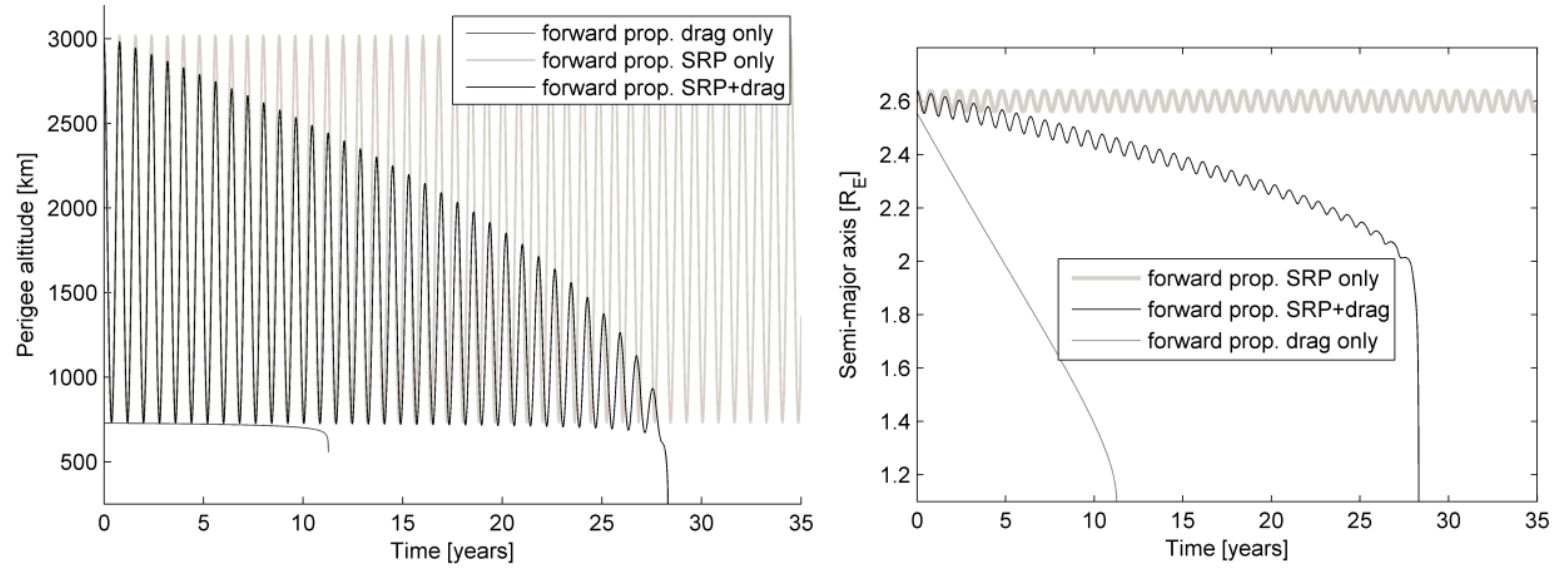

a)

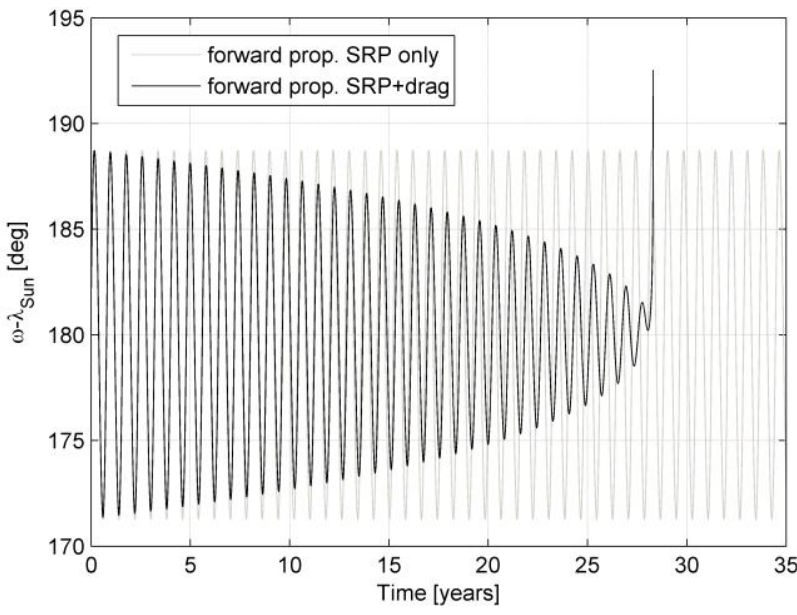

b)

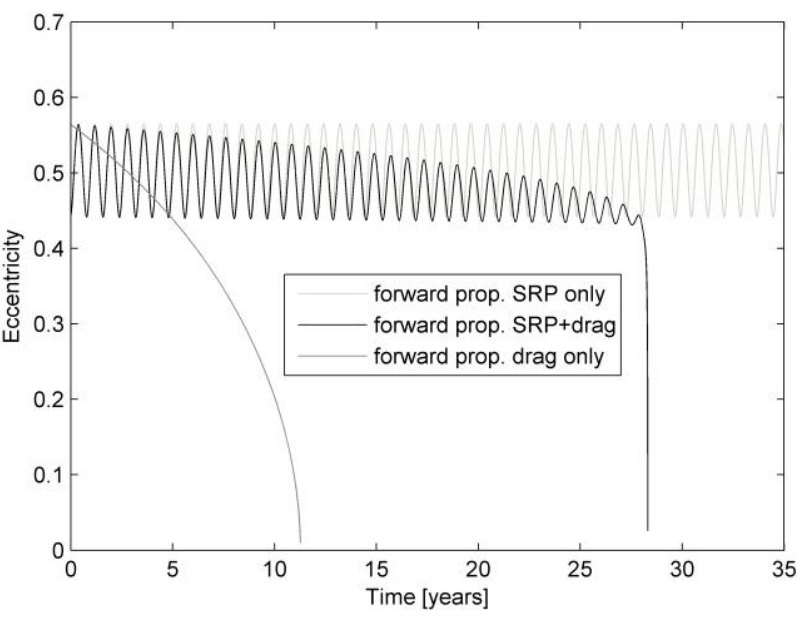

c)

Journal of Journal of Guidance, Control and Dynamics d) 
Fig. 17 Evolution of the Keplerian elements for SpaceChip 3, under the effect of SRP and drag (black line), drag only (gray line), and SRP only (light gray line). a) Perigee altitude, b) semi-major axis in Earth radii, c) angular displacement with respect to the Sun direction, $\omega-\lambda_{\text {Sun }}$, and d) eccentricity.

The orbit evolution analyzed for the selected solution in Fig. 15-Fig. 17 can be reproduced selecting any point straddling the bifurcation region of the long-lived orbits graph (see Fig. 10). This procedure is shown in Fig. 18; different vectors of initial conditions kep $_{L L}$ belonging to the branches Eq. (18) and Eq. (17) of the long-lived orbits graph are selected, and ordered in increasing eccentricity. These sets of orbital elements are indicated in Fig. 18a with a 'o' symbol and gray scale. Starting from those points, the forward and backward propagation of the SpaceChip orbit, under the effect of SRP and drag can be seen. The stopping criterion for the forward integration is defined by Eq. (20), whereas the stopping criterion for the backward integration is defined by Eq. (21), with $h_{p \text {,up }}=8000 \mathrm{~km}$ for this simulation. The first two solutions represented in Fig. 18 (darker gray lines) show the same behavior of the solutions in Fig. 11: the backward propagation from the initial condition vectors $k_{L L} p_{L L}$ terminates without performing any librational loop, because the stopping criterion $e \geq 0.8$ or $h_{p} \leq 250 \mathrm{~km}$ is encountered (see the '+' symbol in Fig. 18d).

The third solution portrayed in Fig. 18, instead, performs a librational loop in the backward evolution around the equilibrium line Eq. (17), until meeting the stopping condition $h_{p} \geq h_{p \text {, up }}$ (see the '+' symbol in Fig. 18d). Fig. 18d highlights that the orbit evolution analyzed in Section V.A (see Fig. 11) and Section V.B (see Fig. 15) do not represent a different behavior. In fact, they can be seen as a continuation with increasing values of the eccentricity of the initial condition vector $k e p_{L L}$ selected on the long-lived orbits graph. The higher the value of eccentricity of the vector $k e p_{L L}$ (we are selecting as initial condition vectors points belonging to the branch Eq. (18) and Eq. (17), sorted in ascending order based on the value of the eccentricity), the higher number of librational loops appears in the backward propagation, and the smaller the radius of the cone that the trajectory will perform in the phase-space around the solution set of stable equilibrium Eq. (17), as can be seen in Fig. 18a. In other terms, the orbit evolution identified by vectors $k e p_{L L}$ with higher values of eccentricity will be bounded within the cone described by the trajectory evolution identified by the vector of initial conditions $k e p_{L L}$ at the edges of the bifurcation region. This means, furthermore, that if we select any vector of initial conditions inside this bifurcation cone (which represent the boundary), the long-term evolution will be enclosed in the cone itself, and the motion will be librational and 
progressively decaying. This is highlighted in Fig. 18b, where an initial condition vector is chosen inside the cone, and the trajectory is propagated forward in time (black line). Moreover, it is interesting to note that all these solutions evolve towards their end-of-life through the bifurcation region, which can be seen (as already pointed out for Fig. 15) as a sink for all librational-decaying trajectories which generate at higher values of the semi-major axis and eccentricities inside the bifurcation cone. Analogously, as shown in Section V.A (see Fig. 11), all the trajectories generated on the branch Eq. (18) $\left(\Delta a_{2 \pi}=0\right.$ and $\left.\Delta \omega_{2 \pi}=\Delta \lambda_{\text {Sun, } 2 \pi}\right)$, or the branch Eq. (19) ( $\Delta e_{2 \pi}=0$ and $\left.\Delta \omega_{2 \pi}=\Delta \lambda_{\text {Sun, } 2 \pi}\right)$ will evolve towards their end-of-life within the region of $\omega-\lambda_{\text {Sun }}$ bounded by the two branches.

Going back to the spiral trajectories shown in Fig. 18a, the higher the value of the eccentricity in the vector $k e p_{L L}$, the higher number of librational loops the spacecraft will perform from an upper value of the perigee altitude, until the final decay. As a consequence, the orbit lifetime from a fixed altitude to final decay is higher, as shown in Fig. 19. Fig. 19 shows the lifetime of the orbit, in logarithmic scale, with the eccentricity of the initial condition vector $k e p_{L L}$ identifying each trajectory. By comparing different spiral trajectories in Fig. 18a, it can be seen that when the trajectory arc in the phase-space between two consecutive librational loops (due to the effect of $\mathrm{SRP}$ ) is more extended, the influence of drag is higher on that arc, thus determining the satellite's lifetime.

For the same reason we can infer that if we select different initial conditions at a certain fixed semi-major axis, with different values of eccentricity, the orbit lifetime will be higher as the initial eccentricity will be chosen close to the equilibrium eccentricity for that value of semi-major axis and it will decrease going in a radial direction from the equilibrium value of the eccentricity (see Fig. 20 and Fig. 21). In fact, at a fixed semi-major axis, the smaller the radius of the librational loop, the lower is the effect of drag and it is limited to a smaller part of the librational loop. In the limit, when a vector $k e p_{L L}$ is chosen along the solution set of system Eq. (17) (i.e., the radius of the librational loop is zero) with a sufficiently high value of semi-major axis, the orbit lifetime will be infinite, as the spacecraft is stable in that position, under the influence of SRP and the non-conservative effect of drag can be neglected. These solutions (without considering Earth's shadow) were found by McInnes et al. for a solar sail mission application [22].

The decrease of the orbit lifetime is clearly a function of the increasing influence of atmospheric drag as can be seen in Fig. 22 as expected. For this test, different initial conditions have been selected with the same eccentricity, $\omega-\lambda_{\text {Sun }}$ and decreasing values of perigee altitude; those initial condition are indicated in Fig. 20 with a '+' symbol 
and gray color scale. The trajectory has been propagated forward in time, under the influence of SRP and drag (continuous line) and drag-only (dashed line) and is represented in Fig. 20. Fig. 21a and Fig. 21b show the time evolution of the perigee altitude and the semi-major axis for the different trajectories, while Fig. 22 shows the orbit lifetime, as a function of the initial perigee altitude of the orbit propagation.
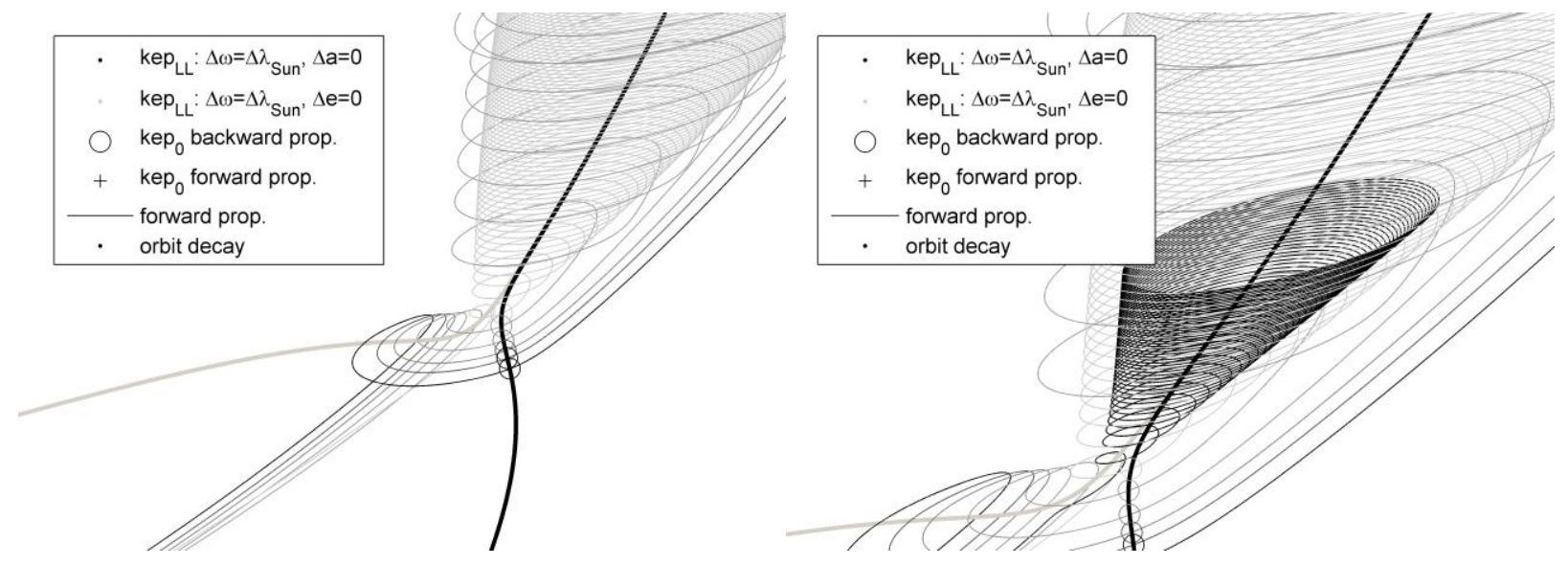

a)

b)
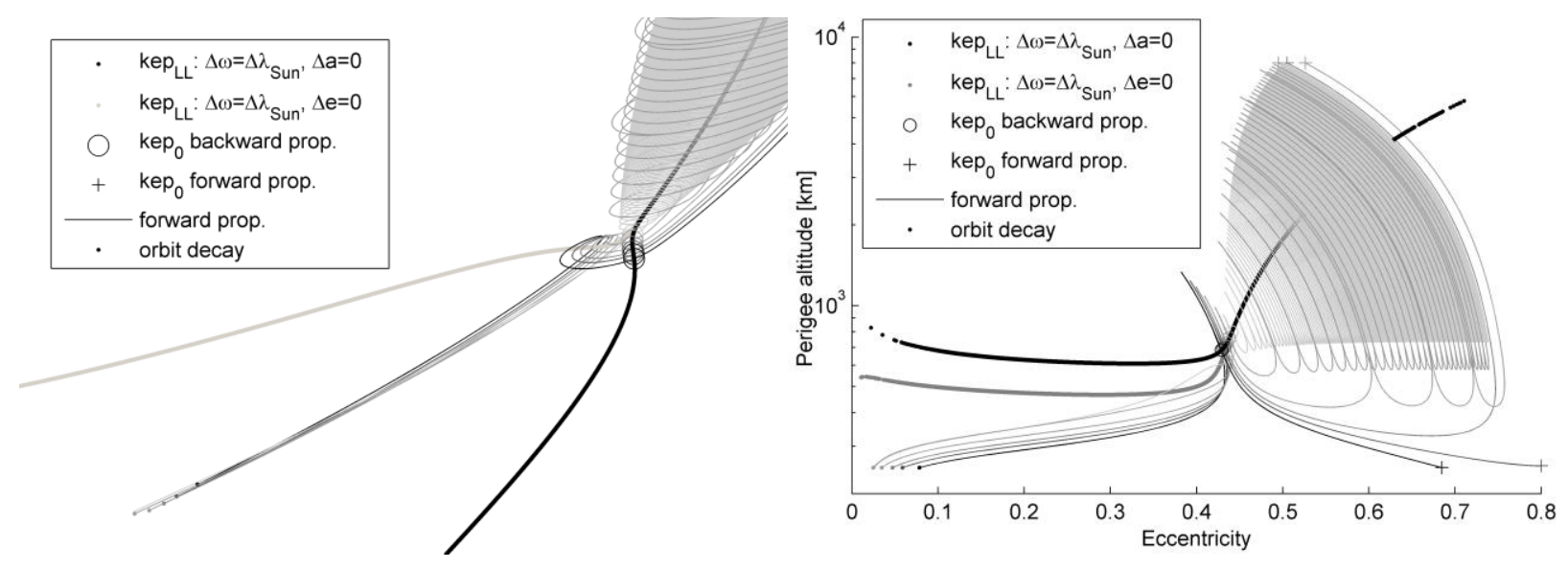

c)

d)

Fig. 18 Long-term orbit evolution for SpaceChip 3 from set of vectors of initial conditions (differentiated with a gray color scale) on the bifurcation region under the effect of SRP and drag: a) zoom on the points selected on the long-lived orbits graph, b) trajectory enclosed in the bifurcation cone, c) zoom on the decay phase, and d) 2D view of the solutions in eccentricity - perigee altitude. The axes of the graph a, b, $\mathbf{c}$ are eccentricity, $\omega-\lambda_{\text {Sun }}$, and semi-major axis. 


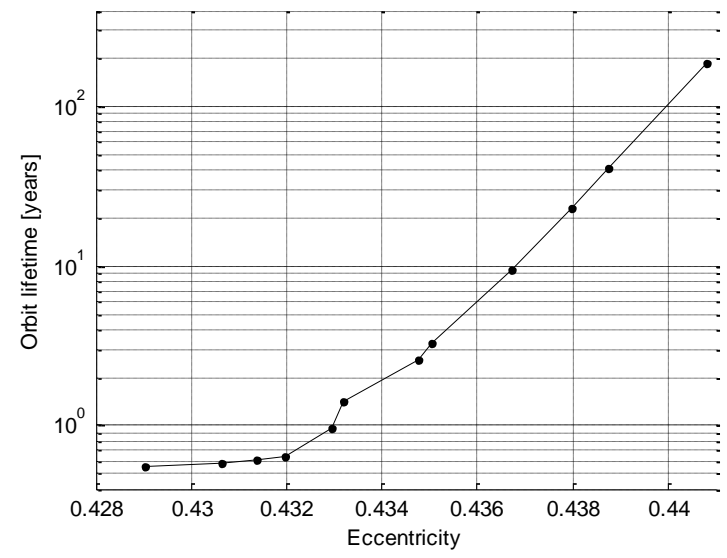

Fig. 19 Orbit lifetime of the solutions for SpaceChip 3 shown in Fig. 18.
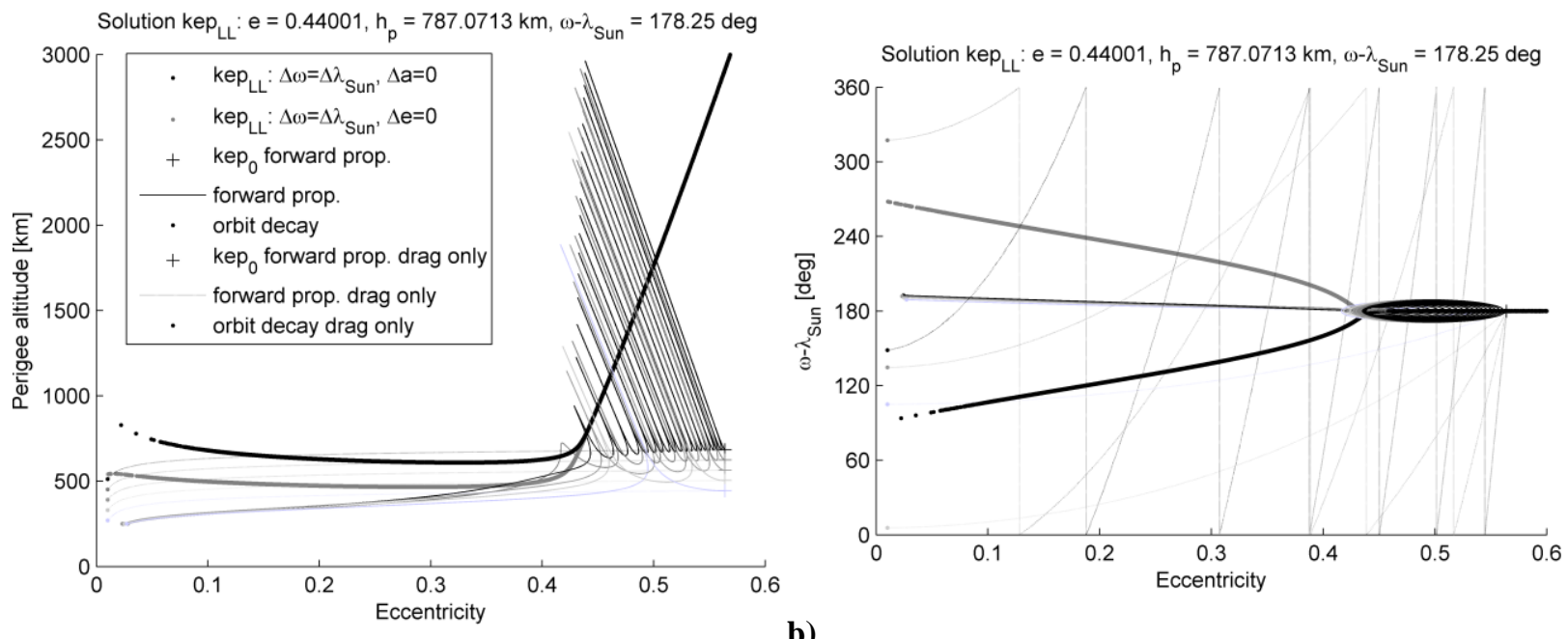

a)

b)

Fig. 20 Long-term orbit evolution for SpaceChip 3 from set of vectors of initial conditions with different perigee altitude (gray color scale), under the influence of SRP and drag (continuous line) and drag only (dashed line). a) 2D view in eccentricity - perigee altitude, and b) 2D view in eccentricity - $\omega$ $\lambda_{\text {Sun }}$. In the drag-only case the motion is rotational. 


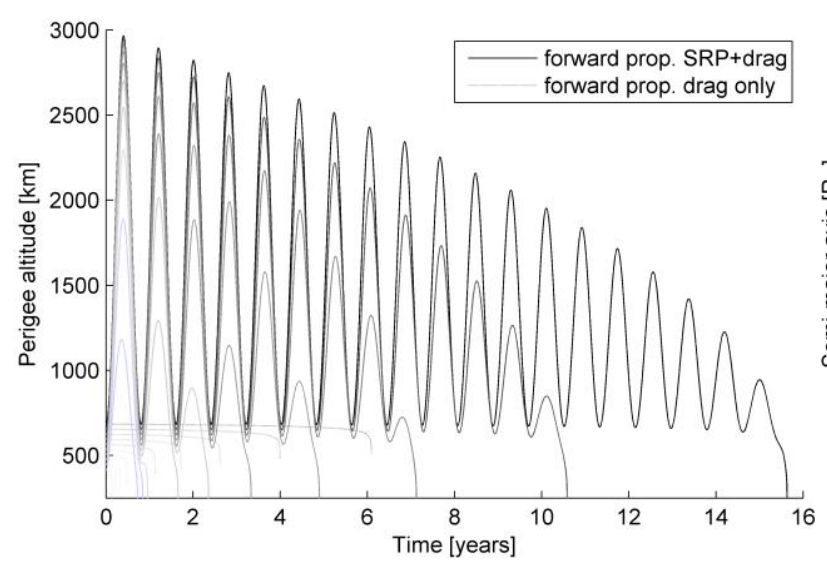

a)

Fig. 21 Evolution of the Keplerian elements for the solutions in Fig. 20. The continuous line represents the SRP and drag scenario, the dashed line represents drag-only scenario. a) Perigee altitude, b) semimajor axis in Earth radii.

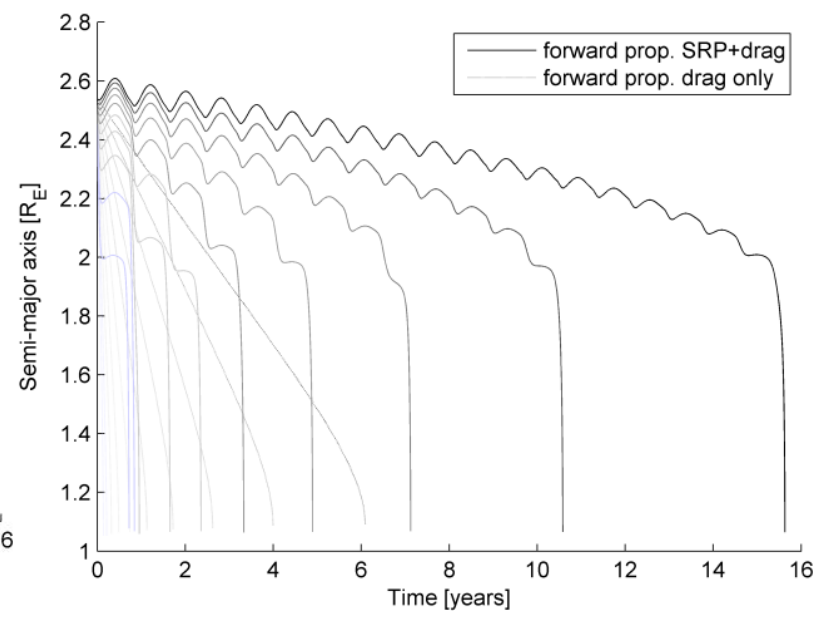

b)

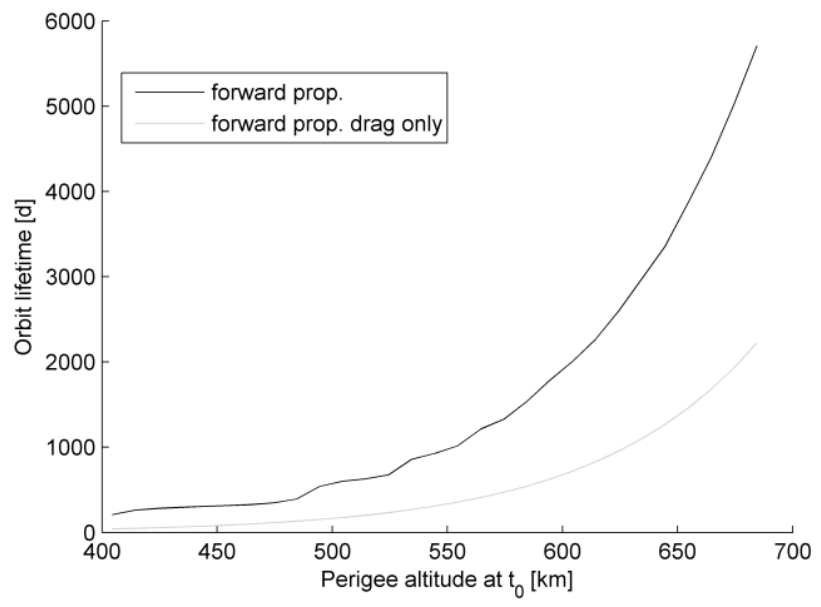

Fig. 22 Orbit lifetime of the solutions for SpaceChip 3 shown in Fig. 20. The black line is the SRP and drag case, the gray line is the drag-only case.

Finally, we point out that there exists a wider set of initial conditions for which the long-term evolution is characterized by a librational and progressively decaying motion. Those orbits can be identified as those which intersect the surface $\Delta \omega_{2 \pi}=\Delta \lambda_{\text {Sun, } 2 \pi}$, as shown in Fig. 23 and Fig. 24 (in correspondence to the intersection with the surface $\Delta \omega_{2 \pi}=\Delta \lambda_{\text {Sun, } 2 \pi}$ the partial derivative with respect to $\omega-\lambda_{\text {Sun }}$ is zero as can be seen in Fig. 24b). In order to identify those orbits, the corresponding initial condition was chosen with $\omega-\lambda_{\text {Sun }}=\pi$ and, fixing a value of semimajor axis, the eccentricity must be lower that the eccentricity for which Eq. (17) is satisfied. These points are 
represented with a '+' symbol in Fig. 23 and Fig. 24. Starting from these condition the forward and backward propagation were performed, to show the long-term behavior in the phase-space. As noted, these orbits are characterized by a librational and progressively decaying motion, however the decay phase will not be enclosed in the bifurcation cone shown in Fig. 18b. All these trajectories, similarly to the trajectories presented in Fig. 11, present an elbow in the phase-space in correspondence of meeting the surface $\Delta a_{2 \pi}=0$ or $\Delta e_{2 \pi}=0$.

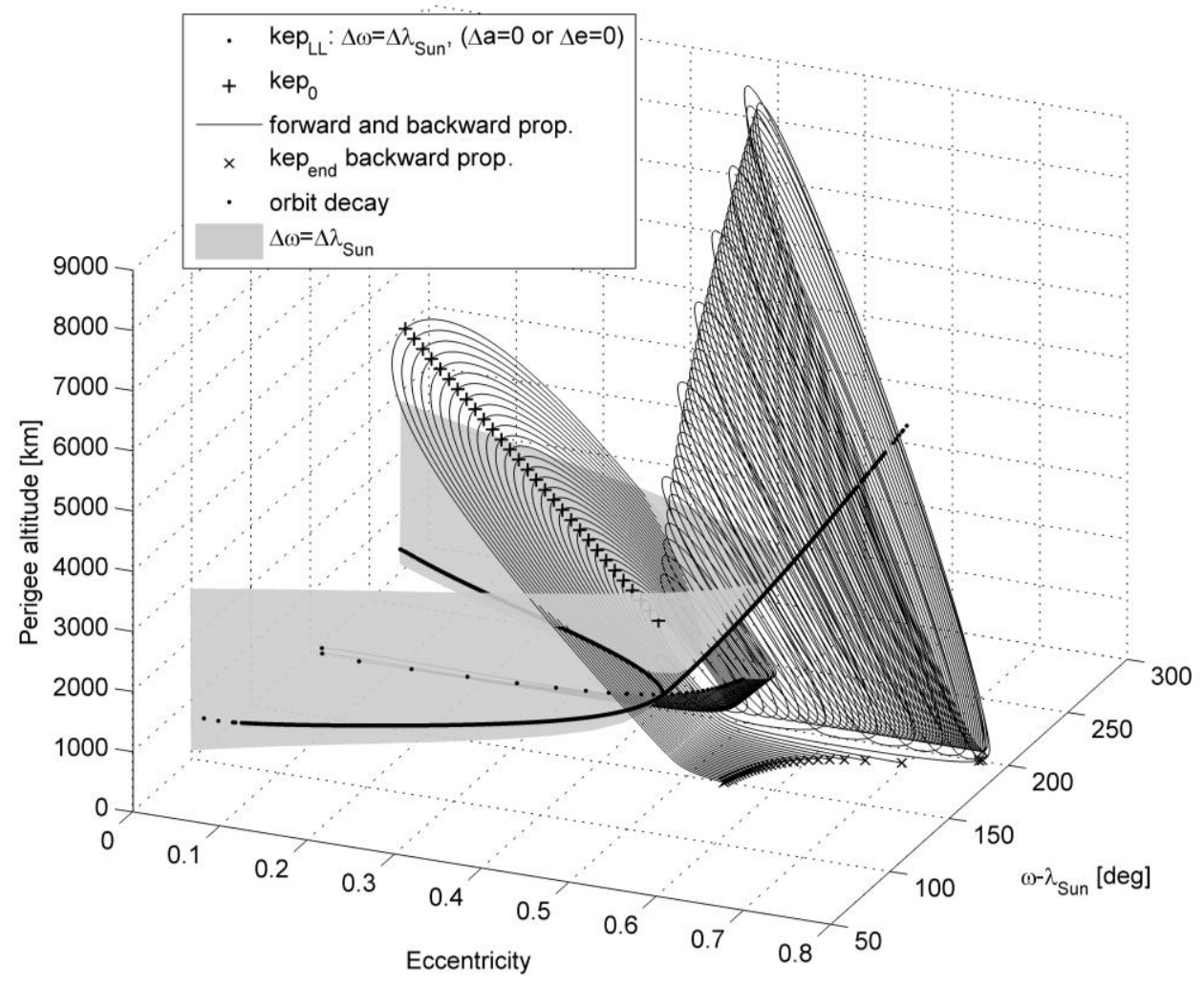

Fig. 23 Long-term orbit evolution for SpaceChip 3 for condition with librational and progressively decaying motion. 

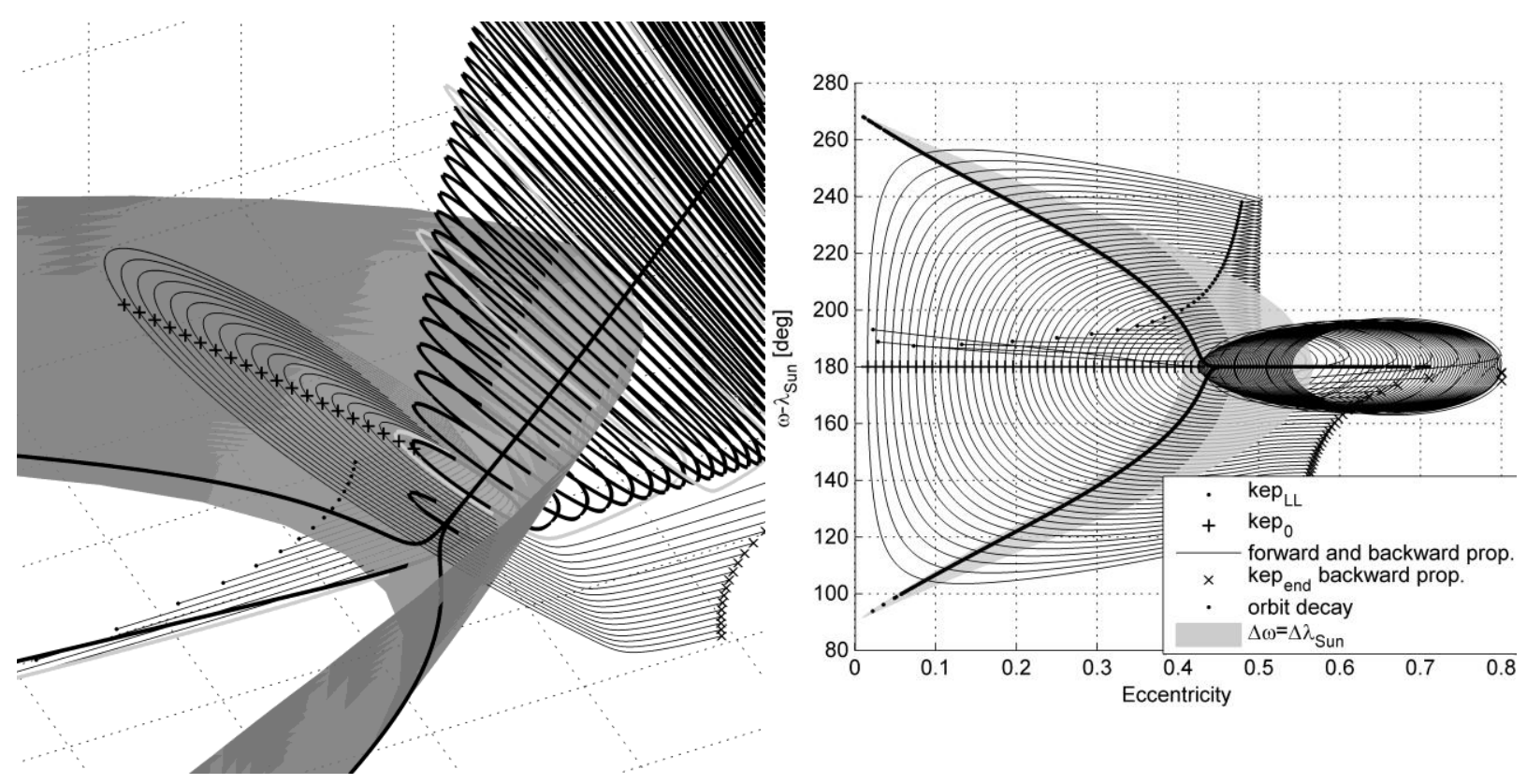

a)

b)

Fig. 24 Long-term orbit evolution for SpaceChip 3 for condition with librational and progressively decaying motion. a) Zoom on the bifurcation region, and b) $2 D$ view in eccentricity $-\omega-\lambda_{\text {Sun. }}$.

The higher the area-to-mass ratio, the more extended is the region of the phase-space domain where the motion under SPR and drag is librational, thus enlarging the sun-synchronous mission possibilities. Outside this region, instead, the motion is rotational (where $\omega-\lambda_{\text {sun }}$ continues to decrease). For a more extensive definition of rotational motion, see Ref. [23].

\section{Mission applications}

McInnes at al. [22] showed that a solar sail with a characteristic acceleration of $0.138 \mathrm{~mm} / \mathrm{s}^{2}$ can be used for a geomagnetic tail mission on a 10 X 30 Earth radii orbit, to artificially precess the apse line in a Sun-synchronous manner. Oyama et al. [23] extended the analysis in the phase-space to study the global behavior of the solar sail orbits around the Earth. With respect to McInnes at al., a more extended useful region of the orbital element space was identified to increase the scientific return of the mission. In this paper it was shown that the Sun-synchronous apse-line precession can be artificially obtained with a SpaceChip device of $1 \mathrm{~cm}^{2}$ area and different thickness values, at different technology readiness levels of current nano-fabrication technologies. This concept can be adapted to enhance the return of a GEOSAIL type mission. A swarm of SpaceChips can be used as distributed nodes 
of a network in the useful region of the phase-space, to obtain a spatial and temporal map of the geomagnetic tail, similar to the concept of the Kilo-Satellite constellation proposed by Petschek et al. [35].

In general, the coupled effect of atmospheric drag and solar radiation pressure, with asymmetry due to eclipses can be exploited to extend the orbit lifetime of a swarm of such devices and to assess and design the disposal of the devices at the end of mission. Notably, the short lifetime of high area-to-mass spacecraft can be greatly extended (and indeed selected) through the interaction of energy gain from asymmetric solar radiation pressure and energy dissipation due to drag. Future missions for swarm of SpaceChips were proposed for the study of the upper layers of

the Earth atmosphere [8]. Moreover, the value of $c_{R}, c_{D}$ and $\frac{A}{m}$ can be engineered for selecting different conditions for long-lived orbits in the phase space. The modulation of SRP on the spacecraft through the change of its reflectivity coefficient was also assessed as a possible means for orbit control [33, 36].

\section{Conclusions}

This paper analyzed the orbital dynamics of future 'smart dust' devices under the influence of solar radiation pressure and atmospheric drag. Their high area-to-mass ratio, with respect to conventional spacecraft, allows the exploitation of the disturbances on the Keplerian motion as a passive method to control the orbit evolution without the use of active orbit control. The secular and long-period changes of the Keplerian elements were computed through semi-analytic formulae. The initial conditions for long-lived orbits for SpaceChips were determined by exploiting energy gain from asymmetric solar radiation pressure to offset the effect of energy dissipation due to atmospheric drag. Through the long-term propagation of the orbit evolution we identified regions of the orbit element phase-space in which the motion of the spacecraft is librational and progressively decaying due to nonconservative energy losses due to drag. Thus, the lifetime of high area-to-mass spacecraft can be greatly extended (and indeed selected) through the exploitation of asymmetric solar radiation pressure, and the effect of drag can be exploited to obtain a fast decay of such 'smart dust' devices in the terminal phase of the mission, dealing with issues related to end-of-life disposal and the creation of long-lived space debris from swarm of devices. For higher values of perigee altitude, a set of solutions was identified for which the orbit is in equilibrium under the effect of SRP only, and the influence of drag is negligible. These families of equilibrium and librational orbits are proposed as baseline for future swarm missions for applications such as geomagnetic tail observation and radio astronomical 
measurement. The high area-to-mass ratio allows an enlargement of the useful region of the orbital element space for increasing science return.

\section{Acknowledgments}

This work was funded by the European Research Council, as part of project VISIONSPACE (227571).

\section{References}

[1] Warneke, B. A. and Pister, K. S. J., "MEMS for Distributed Wireless Sensor Networks," Proceedings of the $9^{\text {th }}$ International Conference on Electronics, Circuits and Systems, Vol. 1, 2002, pp. 291-294.

[2] Vladimirova, T., Xiaofeng, W. and Bridges, C. P., "Development of a Satellite Sensor Network for Future Space Missions," Proceedings of the Aerospace Conference, 2008 IEEE, 2008, pp. 1-10.

[3] Barnhart, D. J., Vladimirova, T. and Sweeting, M. N., "Very-Small-Satellite Design for Distributed Space Missions," Journal of Spacecraft and Rockets, Vol. 44, No. 6, 2007, pp. 1294-1306. doi: 10.2514/1.28678

[4] Miller, L. M., "MEMS for Space Applications," Symposium on Design, Test and Microfabrication of MEMS/MOEMS - Review Paper, Paris, France, 1999, pp. 1-11.

[5] Janson, S. W., "Silicon Satellites: Picosats Nanonats, and Microsats," Proceedings of the International Conference on Integrated Micro/Nanotechnology for Space Applications, The Aerospace Press, Houston, Texas, 1995.

[6] Warneke, B., Last, M., Liebowitz, B. and Pister, K. S. J., "Smart Dust: Communicating with a CubicMillimeter Computer," Computer, Vol. 34, No. 1, 2001, pp. 44-51.

[7] Atchison, J. A. and Peck, M. A., "A Passive, Sun-Pointing, Millimeter-Scale Solar Sail," Acta Astronautica, Vol. 67, No. 1-2, 2010, pp. 108-121. doi: 10.1016/j.actaastro.2009.12.008

[8] Colombo, C. and McInnes, R. C., "Orbit Design for Future Spacechip Swarm Missions," 61 ${ }^{\text {st }}$ International Astronautical Congress, Prague, 2010, IAC-10-C1.8.2.

[9] Shapiro, I. I., Jones, H. M. and Perkins, C. W., "Orbital Properties of the West Ford Dipole Belt," Proceedings of the IEEE, Vol. 52, No. 5, 1964, pp. 469-518.

[10] Hamilton, D. P. and Krivov, A. V., "Circumplanetary Dust Dynamics: Effects of Solar Gravity, Radiation Pressure, Planetary Oblateness, and Electromagnetism,” Icarus, Vol. 123, No. 2, 1996, pp. 503-523. doi: 10.1006/icar.1996.0175

[11] Greenberg, R. and Brahic, A. (eds.), Planetary Rings, Space Science Series. University of Arizona Press, Tucson, 1984.

[12] Grotta-Ragazzo, C., Kulesza, M. and Salomao, P. A. S., "Equatorial Dynamics of Charged Particles in Planetary Magnetospheres," Physica D: Nonlinear Phenomena, Vol. 225, No. 2, 2007, pp. 169-183. doi: 10.1016/j.physd.2006.10.009

[13] Gor'kavyi, N. N., Ozernoy, L. M. and Mather, J. C., "A New Approach to Dynamical Evolution of Interplanetary Dust,” Astrophysical Journal, Vol. 474, No. 1, 1997, pp. 496-496. doi: 10.1086/303440

[14] Howard, J. E., Horanyi, M. and Stewart, G. R., "Global Dynamics of Charged Dust Particles in Planetary Magnetospheres," Physical Review Letters, Vol. 83, No. 20, 1999, pp. 3993-3996. doi: 10.1103/PhysRevLett.83.3993

[15] Schildknecht, T., Musci, R. and Flohrer, T., "Properties of the High Area-to-Mass Ratio Space Debris Population at High Altitudes," Advances in Space Research, Vol. 41, No. 7, 2008, pp. 1039-1045. doi: 10.1016/j.asr.2007.01.045

[16] Anselmo, L. and Pardini, C., "Long-Term Dynamical Evolution of High Area-to-Mass Ratio Debris Released into High Earth Orbits," Acta Astronautica, Vol. 67, No. 1-2, 2010, pp. 204-216. doi: 10.1016/j.actaastro.2009.10.017

[17] Shapiro, I. I. and Jones, H. M., "Perturbations of the Orbit of the Echo Balloon," Science, Vol. 132, No. 3438, 1960, pp. 1484-1486. doi: 10.1126/science.132.3438.1484

Journal of Journal of Guidance, Control and Dynamics 
[18] Musen, P., Bryant, R. and Bailie, A., "Perturbations in Perigee Height of Vanguard I," Science, Vol. 131, No. 3404, 1960, pp. 935-936. doi: 10.1126/science.131.3404.935

[19] Harwood, N. M. and Swinerd, G. G., "Long-Periodic and Secular Perturbations to the Orbits of Explorer 19 and Lageos Due to Direct Solar Radiation Pressure," Celestial Mechanics and Dynamical Astronomy, Vol. 62, No. 1, 1995, pp. 81-92. doi: 10.1007/BF00692070

[20] McInnes, C. R., Solar Sailing: Technology, Dynamics and Mission Applications, Springer-Praxis Books in Astronautical Engineering, Springer-Verlag, Berlin, 1999, Ch. 2.

[21] Kawaguchi, J. i., Mimasu, Y., Mori, O., Funase, R., Yamamoto, T. and Tsuda, Y., "IKAROS - Ready for Lift-Off as the World's First Solar Sail Demonstration in Interplanetary Space," Proceedings of the $60^{\text {th }}$ International Astronautical Congress, IAC 2009, Daejeon, Korea, 2009, IAC-09-D1.1.3.

[22] McInnes, C. R., Macdonald, M., Angelopolous, V. and Alexander, D., "Geosail: Exploring the Geomagnetic Tail Using a Small Solar Sail," Journal of Spacecraft and Rockets, Vol. 38, No. 4, 2001, pp. 622-629.

[23] Oyama, T., Yamakawa, H. and Omura, Y., "Orbital Dynamics of Solar Sails for Geomagnetic Tail Exploration," Journal of Guidance, Control and Dynamics, Vol. 45, No. 2, 2008, pp. 316-323. doi: $10.2514 / 1.31274$

[24] De Juan Ovelar, M., Llorens, J. M., Yam, C. H. and Izzo, D., "Transport of Nanoparticles in the Interplanetary Medium," 60 $0^{\text {th }}$ International Astronautical Congress, Daejeon, Republic of Korea, 2009.

[25] Vallado, D. A., Fundamentals of Astrodynamics and Applications, Third Edition, Space Technology Library, New York, 2007, Sections 8.6.2, 8.6.4 and 9.6.2.

[26] Fortescue, P., Stark, J. and Swinerd, G. (eds.), Spacecraft System Engineering, Third Edition ed. Wiley, Chichester, 2003, pp. 93-95.

[27] King-Hele, D., Theory of Satellite Orbits in an Atmosphere, Butterworths, London, 1964.

[28] Blitzer, L., "Handbook of Orbital Perturbations," University of Arizona, 1970.

[29] Abramowitz, M. and Stegun, I. A., Handbook of Mathematical Functions, Applied Math. Series \#55, Dover Publications, 1965, Ch. 9.

[30] Colombo, C. and McInnes, C., "Orbital Dynamics of Earth-Orbiting 'Smart Dust' Spacecraft under the Effects of Solar Radiation Pressure and Aerodynamic Drag," AIAA/AAS Astrodynamics Specialist Conference 2010, Toronto, Canada, 2010, AIAA 2010-7656.

[31] Coleman, T. F. and Li, Y., "On the Convergence of Interior-Reflective Newton Methods for Nonlinear Minimization Subject to Bounds," Mathematical Programming, Vol. 67, No. 1, 1994, pp. 189-224.

[32] Coleman, T. F. and Li, Y., "An Interior Trust Region Approach for Nonlinear Minimization Subject to Bounds," SIAM Journal on Optimization, Vol. 6, No. 2, 1996, pp. 418-445.

[33] Colombo, C., Lücking, C. M. and McInnes, C. R., "Orbit Evolution, Maintenance and Disposal of Spacechip Swarms," $6^{\text {th }}$ International Workshop on Satellite Constellation and Formation Flying (IWSCFF 2010), Taipei, Taiwan, 2010, IWSCFF-Paper-2010-2-2.

[34] Dormand, J. R. and Prince, P. J., "A Family of Embedded Runge-Kutta Formulae," Journal of computational and applied mathematics, Vol. 6, No. 1, 1980, pp. 19-26.

[35] Petschek, H. E., Rayburn, C., Sheldon, R., Vickers, J., Bellino, M., Bevis, G. and Spence, H. E., Science Closure and Enabling Technologies for Constellation Class Missions, Library of Congress Cataloging-inPublication Data, University of California, Berkeley, 1998, pp. 51-57.

[36] Lücking, C. M., Colombo, C. and McInnes, C., "Orbit Control of High Area-to-Mass Ratio Spacecraft Using Electrochromic Coating," 61 ${ }^{\text {st }}$ International Astronautical Congress, Prague, 2010, IAC-10-C1.2.7. 